University of Louisville

ThinkIR: The University of Louisville's Institutional Repository

Electronic Theses and Dissertations

$12-2017$

\title{
Targeting the major regulator of mitosis.
}

J. Mason Hoffman

University of Louisville

Follow this and additional works at: https://ir.library.louisville.edu/etd

Part of the Medicine and Health Sciences Commons

\section{Recommended Citation}

Hoffman, J. Mason, "Targeting the major regulator of mitosis." (2017). Electronic Theses and Dissertations. Paper 2879.

https://doi.org/10.18297/etd/2879

This Master's Thesis is brought to you for free and open access by ThinkIR: The University of Louisville's Institutional Repository. It has been accepted for inclusion in Electronic Theses and Dissertations by an authorized administrator of ThinkIR: The University of Louisville's Institutional Repository. This title appears here courtesy of the author, who has retained all other copyrights. For more information, please contact thinkir@louisville.edu. 


\title{
TARGETING THE MAJOR REGULATOR OF MITOSIS
}

\author{
By \\ J. Mason Hoffman \\ A Thesis Submitted \\ to the Faculty of the School \\ of Medicine of the University of Louisville \\ in Partial Fulfillment of the Requirements \\ for the Degree of \\ Masters of Science \\ Pharmacology and Toxicology \\ Department of Pharmacology and Toxicology \\ University of Louisville \\ Louisville, Kentucky
}

December 2017 

TARGETING THE MAJOR REGULATOR OF MITOSIS

\author{
By \\ J. Mason Hoffman \\ B.S., Sewanee: The University of the South, 2014
}

A Thesis Approved on

July 27,1017

by the following Thesis Committee:

J. Christopher States, Ph.D.

Chendil Damodaran, Ph.D.

Chi Li, Ph.D.

John O. Trent, Ph.D.

John P. Wise, Sr., Ph.D. 


\section{ABSTRACT \\ TARGETING THE MAJOR REGULATOR OF MITOSIS \\ J. Mason Hoffman}

July 27,2017

Mitosis-inhibiting chemotherapeutics (e.g. taxanes) are frequently used to treat multiple cancer types. Recently, there has been much concern about the limited success of these drugs due to resistance and a lack of molecular targets. Thus, there is high demand for new drugs with diverse cellular targets. Targeting the regulators of mitosis is a promising approach. The anaphase promoting complex/cyclosome (APC/C) is an E3 ubiquitin ligase that controls cell cycle progression at multiple points. The interaction of ANAPC2 and ANAPC11, catalytic core subunits, is necessary for APC/C function. An in silico approach was used to identify compounds predicted to prevent assembly of ANAPC2 and ANAPC11, causing APC/C inhibition and mitotic arrest. Several of the predicted compounds possess cytotoxic properties in multiple cancer cell types. These hit compounds induce mitotic arrest and cell death in malignant, but not nonmalignant cells. Additionally, hit compounds are effective in taxane-resistant cells. 


\section{TABLE OF CONTENTS}

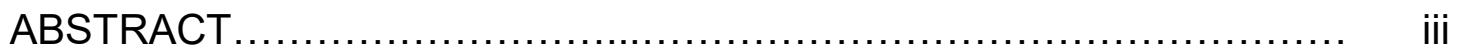

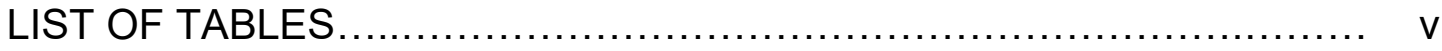

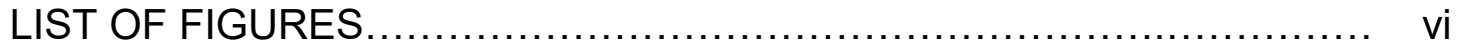

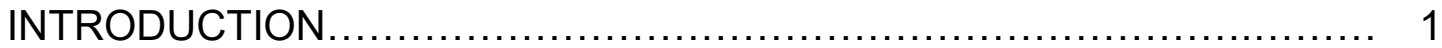

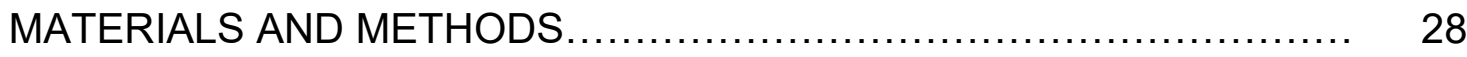

Cell Culture....................................................... 28

AlamarBlue Assays................................................ 29

Colony Forming Assays......................................... $\quad 30$

Mitotic Index Analysis............................................ 31

G1/S Synchronization........................................ 32

Western Blotting ................................................. 33

Flow Cytometry................................................... $\quad 35$

Apoptosis Assays .................................................. $\quad 36$

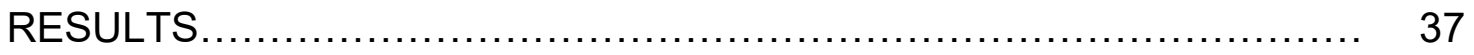

AlamarBlue Screening in Multiple Cell Types....................... 37

Colony Forming Assays in Multiple Cell Types...................... $\quad 39$

Mitotic Index Analysis of Multiple Cell Types......................... $\quad 42$

Stability of APC/C Substrates in Synchronized A375 Cells ........... 44

Cell Cycle Effects of Hit Compounds in a SAC-Deficient Melanoma 48

Cell Line.

Apoptosis Assays in Lung and Ovarian Cell Lines................... 51

DISCUSSION........................................................... 53

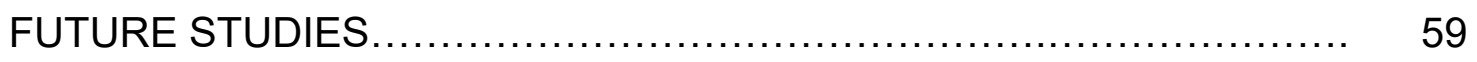

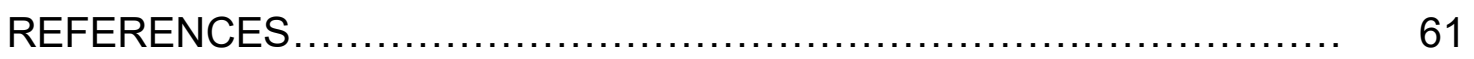

APPENDIX: LIST OF ABBREVIATIONS ............................... 67

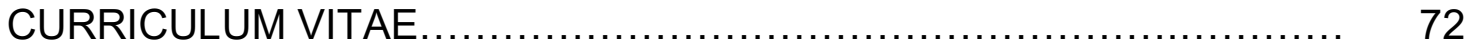




\section{LIST OF TABLES}

Table 1. Reported substrates of each F-box subfamily ................. 10

Table 2. List of reported $A P C / C(C D C 20)$ and $A P C / C(C D H 1)$ targets....................................................... 14 


\section{LIST OF FIGURES}

Figure 1. Outline of protein degradation by the ubiquitin proteasome system

Figure 2. Illustration of the mechanism of the SAC ...................... 18

Figure 3. Models of the ANAPC2:ANAPC11 binding interaction and structures of predicted inhibitors

Figure 4. Hit compounds reduce viability in lung cell lines............... 38

Figure 5. Hit compounds reduce colony forming ability of multiple lung, ovarian, pancreatic, and melanoma cell lines.

Figure 6. Compounds cause cytostasis in non-malignant lung cells.........

Figure 7. Lung and ovarian cells exposed to hit compounds have increased populations in mitosis

Figure 8. APC/C mitotic targets display extended kinetics of degradation when exposed to hit compounds.

Figure 9. Compounds targeting distinct, overlapping regions of ANAPC2 induce cell cycle arrest at different points in SAC-deficient melanoma cells

Figure 10. Levels of active caspase-3/7 are elevated in lung and ovarian cancer cells following exposure to hit compounds

Figure 11. Two regions of ANAPC2 targeted in silico and predicted binding interactions of compounds 3 and 8 . 


\section{INTRODUCTION}

Mitosis-inhibiting chemotherapeutics (e.g. taxanes) are frequently used in combination chemotherapy to treat multiple types of cancer. These are effective because they exploit the mitotic spindle assembly checkpoint (SAC), causing inhibition of the anaphase promoting complex/cyclosome (APC/C), mitotic arrest, and apoptosis. Successful therapy is often hindered by drug-resistant tumors, neurotoxicity, and limited supply. All current mitotic inhibitors, and the majority that are in development, target a single protein: tubulin. Therefore, mutations in tubulin or SAC proteins create cancer cells that are resistant to all current mitotic inhibitors. Thus, there is high demand for new drugs with diverse cellular targets. Rather than targeting tubulin, a promising alternative approach is targeting the proteins that regulate mitosis. The APC/C is an E3 ubiquitin ligase that is vital for progression through mitosis and G1-phase. The APC/C ubiquitinylates its protein targets, which marks them for degradation. Many target proteins of the APC/C have well characterized proliferative or anti-proliferative roles. By carefully orchestrating the degradation of these proteins, the APC/C facilitates the orderly progression of the cell cycle and timing of critical cellular events.

\section{The Ubiquitin-Proteasome System and the Cell Cycle}

The cell cycle is a highly regulated unidirectional sequence of events in which a cell replicates its genetic material and divides into two daughter cells. A 
complex network of proteins is required to coordinate progression through each phase of this process. In eukaryotic cells, the cell cycle is divided into four phases: G1 (gap 1), S (Synthesis, DNA replication), G2 (gap 2), and M (Mitosis, division of cellular components to two daughter cells).

Of particular importance in controlling phase transitions are a family of protein kinases, known as cyclin-dependent kinases (CDKs). CDKs are constitutively-expressed and regulate the activity of their targets by phosphorylation. As their name suggests, they are only active when associated with positive regulatory subunits, known as cyclins [1]. The prototypical cyclins involved in cell cycle control are cyclins A, B, D, and E, each of which associates with different CDKs. Expression of cyclins, unlike CDKs, oscillates during each cell cycle resulting in activation of different CDKs at unique times. Thus, through their control of CDK activity, cyclins are key to promoting cell cycle progression. This pattern of CDK activation allows phosphorylation of key targets involved in DNA replication and mitosis at the appropriate times. Further complicating matters, CDK activity is negatively regulated by post-translational modifications (PTMs, e.g. phosphorylation) and the periodic expression of CDK inhibitors (CDKNs) [2]. A delicate balance between synthesis and degradation of cyclins, CDKNs, and other regulatory proteins is required for cell cycle control [1,3].

Perhaps the most important means of CDK regulation is the periodic degradation of cyclins and CDKNs by ubiquitin-mediated proteolysis $[4,5]$. Ubiquitin is a small, highly-conserved protein that is covalently attached to a target protein through a process called ubiquitinylation. It is the slowest evolving 
protein yet identified, highlighting the conserved importance of its function. Ubiquitinylation, like other PTMs, can alter the stability, structure, localization and function of target proteins $[6,7]$. More specifically, ubiquitinylation is the formation of an isopeptide bond between the C-terminus of ubiquitin and a lysine within a confined domain of the target. Polyubiquitinylation is a modification which contains chains of multiple linked ubiquitins $[6,8,9]$. In terms of cell cycle control, the predominant function of polyubiquitinylation is to mark target proteins, such as cyclins, CDKNs, and other key regulatory proteins, for degradation [10].

While polyubiquitination can have numerous cellular effects, one of the most studied roles of polyubiquitination is in protein degradation. Other functions of ubiquitinylation include enzyme activation, epigenetic modification, receptor internalization, apoptosis, polycomb silencing, and signaling pathways such as tumor necrosis factor, interleukin 1 beta, CD40 ligand, Toll-like receptors, and Nucleotide-binding oligomerization domain-containing protein 2 [11-15]. As would be expected of a system that regulates so many important cellular processes, there is a large and growing list of proteins involved in this process of ubiquitinylation and degradation of target proteins. These proteins comprise what is known as the ubiquitin-proteasome system (UPS). This system of selective, programmed protein degradation ensures the unidirectionality of the cell cycle and the appropriate timing of cell cycle events $[5,10,16]$.

The ubiquitin-proteasome system facilitates the degradation of target proteins by directing them to the $26 \mathrm{~S}$ proteasome, a large multi-subunit complex consisting of multiple proteases $[17,18]$. The core of the proteasome, termed the 
20S proteasome, contains chymotrypsin-like, trypsin-like, and caspase-like enzymatic activities [17]. The $26 \mathrm{~S}$ proteasome is formed by association of two $19 S$ caps with opposite ends of the 20S core [7]. These caps act as regulatory subunits for recognition of ubiquitinylated proteins. Upon recognition, the protein is unfolded in an ATP-dependent manner by the $19 \mathrm{~S}$ subunits and introduced into the inner chamber of the $20 \mathrm{~S}$ subunit for degradation. While rare cases of ubiquitin-independent proteasomal degradation have been documented, most cases rely on signaling via protein ubiquitinylation [17].

Ubiquitinylation of a target protein is facilitated by a 3-step cascade of enzymatic reactions (Figure 1) [7]. The initial step requires a ubiquitin-activating enzyme (E1), which binds to the C-terminal glycine of ubiquitin via an ATPdependent reaction [19]. Following activation, ubiquitin is transferred from the E1 to a ubiquitin-conjugating enzyme (E2), and finally, an E3 ubiquitin ligase transfers ubiquitin from the E2 to the target protein [20]. Multiple rounds of ubiquitinylation occur, allowing the target protein to be recognized and degraded by the $26 S$ proteasome (Figure 1). E3 ubiquitin ligases provide the UPS with a high degree of specificity, which is required for this system to target the correct proteins at the appropriate times $[6,7]$.

E3 ubiquitin ligases, enzymes responsible for the transfer of ubiquitin from an E2 ubiquitin-conjugating enzyme to the target protein, are the largest and 


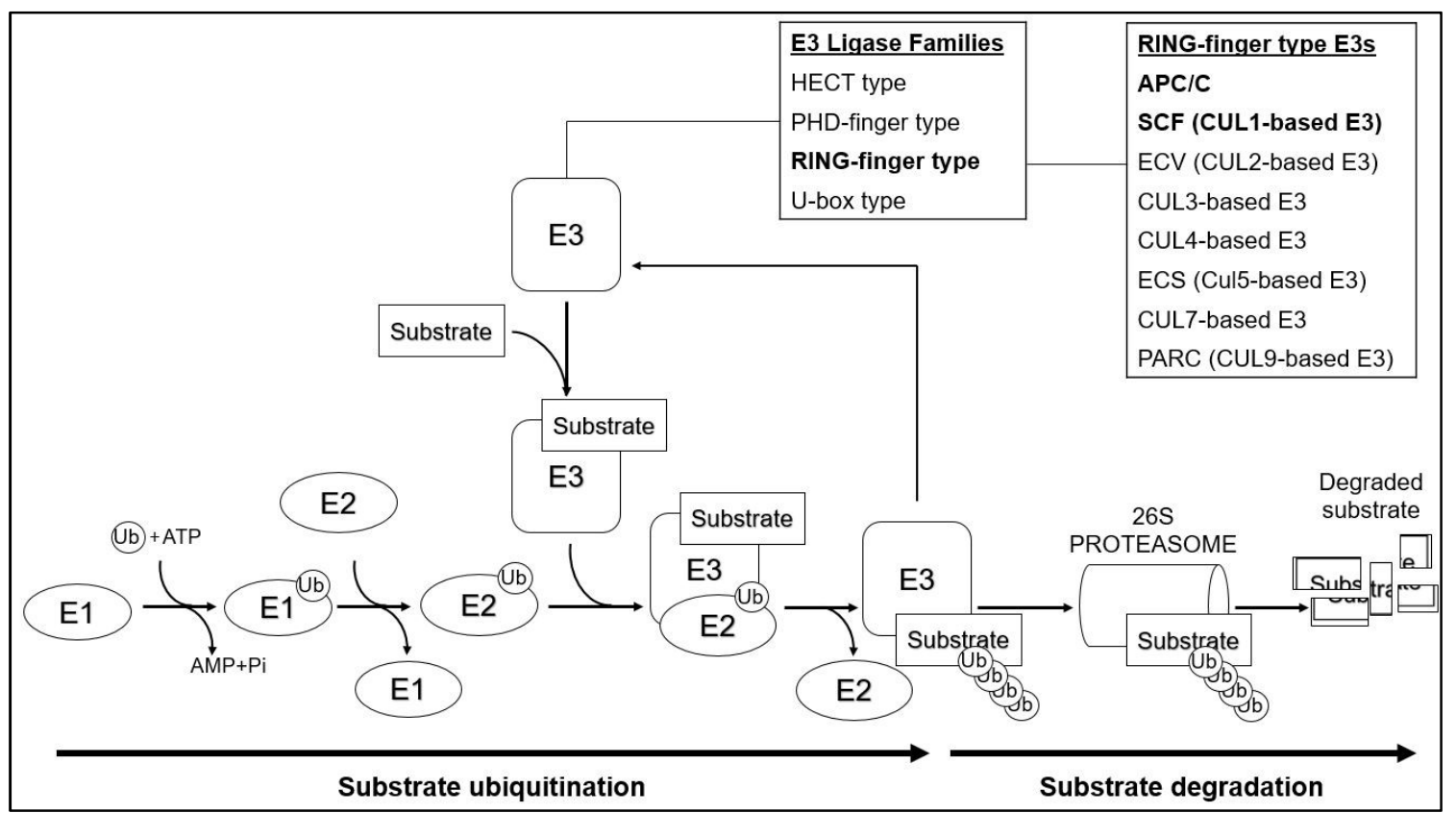

Figure 1. Outline of protein degradation by the ubiquitin proteasome

system. E1 ubiquitin-activating enzymes first covalently attach to ubiquitin via an ATP-dependent reaction. Ubiquitin is then transferred to an E2 ubiquitinconjugating enzyme. An E3 ubiquitin ligase binds its substrate protein and the ubiquitin-bound E2 enzyme and catalyzes the covalent attachment of ubiquitin to the substrate. Multiple rounds of substrate ubiquitinylation lead to the formation of a polyubiquitin chain. The polyubiquitinylated substrate is subsequently recognized and degraded by the $26 \mathrm{~S}$ proteasome. The E3 ubiquitin ligase is now free to repeat the cycle from the beginning. The four main classes of E3 ubiquitin ligases (HECT, PHD-finger, RING-finger, and U-box types) and the members of the RING-finger E3 subfamily (APC/C, SCF, ECV, CUL3-based, CUL4-based, ECS, CUL7-based, and PARC E3s) are listed. The two RING-finger E3s in bold, the $\mathrm{APC} / \mathrm{C}$ and $\mathrm{SCF}$, are both critical for cell cycle regulation. 
most complex members of the E1-E2-E3 cascade [21]. While humans possess only $8 \mathrm{E} 1$ ubiquitin-activating enzymes and $38 \mathrm{E} 2$ ubiquitin-conjugating enzymes, there are more than 600 human E3 ubiquitin ligases [19, 20]. There are four classes of E3 ubiquitin ligases: HECT (homologous to E6-AP C-terminus) domain-containingE3s, U-box domain-containing E3s, PHD (plant homeodomain) finger domain-containing E3s, and RING (really interesting new gene) fingercontaining E3s (Figure 1) [21].

The RING finger E3s are the largest E3 class and are further divided into subclasses (Figure 1). One of these is the cullin-RING ligase (CRL) subclass, which includes two structurally similar protein complexes that are vital to cell cycle regulation: The SKP/cullin/F-box-containing (SCF) complexes and the anaphase promoting complex/cyclosome (APC/C) (Figure 1) [21]. The catalytic cores of these CRLs are highly similar, consisting of a cullin-like protein and a Zn-RING-finger protein [21, 22]. Cullin and Zn-RING-finger proteins are essential for catalyzing the transfer of ubiquitin to the target protein [23]. Additionally, these E3 ubiquitin ligases associate with specific coactivator subunits, which determine substrate specificity [24-26].

E3 ubiquitin ligases, like CDKs, are subject to multiple layers of regulation, including activating and inhibitory PTMs, association with coactivators and inhibitors, and periodic expression of various subunits [24-30]. Additionally, deubiquitinating enzymes (DUBs), proteases that cleave ubiquitin from modified proteins, directly counteract E3 ubiquitin ligases. In humans, over 100 DUBs have been identified, each targeting specific subsets of proteins. DUBs have 
demonstrated roles in many cellular processes, including DNA repair, chromatin remodeling, various signaling pathways, and cell cycle regulation [31]. A study by Huang et. al provided an example of the importance of DUBs in cell cycle regulation [32]. Briefly, they showed that ubiquitin carboxyl-terminal hydrolase 37 (USP37), a DUB that both antagonizes and is a substrate of the APC/C, is important for regulation of S-phase entry [32]. USP37 is fully activated at G1/S by phosphorylation by CDK2/cyclin E or CDK2/cyclin A, allowing it to stabilize cyclin A by deubiquitylation, promoting entry into S-phase. In mitosis, CDK2 inactivation leads to loss of USP37 activity and its degradation as an APC/C substrate. This prevents USP37 from antagonizing the APC/C, allowing timely degradation of key APC/C mitotic substrates, and promoting mitotic progression. Several other examples of DUBs in cell cycle regulation are detailed by Wei et. al [31].

The remainder of this section will focus on the role of CRLs in cell cycle regulation, control of CRL activity, and particularly, the APC/C in regulation of mitosis.

\section{Two Cullin-RING Ligases Control Cell Cycle Progression}

SCF E3 Ubiquitin Ligases are critical components of cell cycle regulation. They are particularly important for regulating entry into S-phase, as well as mitotic entry $[29,33]$. There are three core components of SCF ligases which are invariable: SKP1 (S-phase kinase-associated protein 1), CUL1 (Cullin-1), and RBX1 (RING-box protein 1) [34]. SCF ligases are the largest class of E3 ligases and are responsible for the degradation of an estimated $20 \%$ of ubiquitin- 
proteasome-regulated proteins, including many cell cycle regulatory proteins (Table 1) [6, 7, 35-37].

SCF complexes are classified as CUL1-containing E3 ubiquitin ligases. CUL1 is a cullin protein that functions as a scaffold protein. In addition to CUL1, SCF ubiquitin ligases contain RBX1, a RING-finger protein, and SKP1, an adaptor protein that binds F-box proteins [37]. There are at least 69 human F-box proteins, each with a conserved C-terminal F-box motif for binding SKP1. F-box proteins function as the SCF substrate determining subunit and are divided into three subfamilies based on their C-terminal substrate binding domains: the FBXW (WD40 substrate binding domains), FBXL (leucine-rich repeats (LRR) substrate binding domains), and FBXO (contains other binding domains such as kelch repeats or proline-rich repeats) subfamilies [37-39]. Each F-box protein recognizes a different set of substrates depending on which substrate binding domains they contain. Interestingly, there is an overlap in substrate recognition, allowing one substrate to be targeted by multiple F-box proteins. Because all three subfamilies of F-box proteins are implicated in cell cycle control, these SCF complexes are likely to be ineffective as a chemotherapeutic target due to the redundancy of F-box substrate-targeting.

SCF E3s containing FBXW1 (SCF(FBXW1)), also known as $\beta$ TrCP (beta-transducin repeat containing protein), have been well documented for their role in cell cycle control. FBXW1 is highly homologous to APC/C substrate specificity subunits, CDC20 (cell division cycle 20) and CDH1 (CDC20 homolog 1) [40], which will be discussed in the following paragraphs. SCF(FBXW1) 
complexes have been implicated in the targeting of numerous cell cycle regulators including EMI1/FBXO5 (early mitotic inhibitor 1/ F-box only protein 5), WEE1 (Wee1-like protein kinase), CDC25A (cell division cycle 25A), Cyclin D1, PLK4 (polo-like kinase 4), BORA (protein aurora borealis), and Securin (Table 1) [37]. EMI1 is an inhibitor of the APC/C in S and G2 phase. In prophase, degradation of EMI1 mediated by $\operatorname{SCF}(\mathrm{FBXW} 1)$ is required for activation of the $\mathrm{APC} / \mathrm{C}$ and mitotic progression [41]. This is one of many examples of the complex co-regulatory network between SCF complexes and the APC/C [4, 26, 42]. Similarly, FBXW1-dependent degradation of WEE1 kinase, a negative regulator of mitotic entry, is required for cells to transition from $G 2$ to $M$ [43]. Another SCF(FBXW1) target, PLK4, is a serine/threonine protein kinase critical for centriole duplication [44]. SCF(FBXW1) has been found to target PLK4 for degradation, which prevents centriole amplification and chromosomal instability $[45,46]$. Securin is an inhibitor of the metaphase to anaphase transition in mitosis, and is well-documented as a mitotic substrate of the APC/C [40, 47-53]. However, in response to UV irradiation, securin degradation was found to be mediated by SCF(FBXW1), resulting in cell cycle arrest $[54,55]$. CDC25A degradation is required for entry into S-phase. In response to DNA damage, CDC25A is phosphorylated, allowing its recognition by SCF(FBXW1), leading to delayed S-phase entry $[56,57]$. Another member of the FBXW F-box protein subfamily, FBXW7, targets several key cell cycle regulators. Cyclin E, an activator of CDK2, promotes the G1/S-phase transition and its phosphorylated form is ubiquitinated by SCF(FBXW7) [21, 58]. 
Table 1 Reported substrates of each F-box subfamily.

\begin{tabular}{c|c|c|c} 
F-box subfamily & FBXW & FBXL & FBXO \\
\hline \multirow{7}{*}{ Target proteins } & EMI1/FBXO5 & CDKN1A & Cyclin B \\
& WEE1 & CDKN1B & Cyclin D1 \\
CDC25A & CDKN1C & TP53 \\
& CDC25B & Cyclin E & CHK1 \\
& Cyclin D1 & Cyclin A & CDT1 \\
& Cyclin E & CDH1 & CDT2 \\
& PLK4 & Cyclin D2 & MDM2 \\
& Bora & Cyclin D3 & BRCA1 \\
& Securin & Aurora A & \\
& Aurora A & & \\
& Aurora B & &
\end{tabular}


Aurora kinases $A$ and $B$ are important regulators of mitosis that ensure proper segregation of chromosomes, and both have been identified as SCF(FBXW7) targets [59-62].

FBXL proteins, a 22-member F-box subfamily, are equally important as the FBXW subfamily. Targets of these FBXL proteins include multiple CDKNs, cyclins, and various cell cycle kinases (Table 1) [21, 37]. The most studied member of this subfamily is FBXL1, also called Skp2. Degradation of CDKN1B (Cyclin-dependent Kinase Inhibitor 1B, p27 ${ }^{\text {Kip1}}$ ) is reportedly mediated by FBXL1, a step required for S-phase entry, $[63,64]$. Other negative regulators of the cell cycle, such as the CDK inhibitors CDKN1A (Cyclin-dependent Kinase Inhibitor 1A, p21 ${ }^{\mathrm{CIP} 1}$ ) and CDKN1C (Cyclin-dependent Kinase Inhibitor 1C, p57 ${ }^{\mathrm{Kip} 2}$ ), have been documented as FBXL1 targets $[65,66]$. Degradation of these proteins promotes cell cycle progression in S and G2 phases. Numerous other targets of FBXL-containing SCF complexes have been reported and are reviewed by Nakayama et.al [67] and Zheng et. al [37]. Like the SCF(FBXW) complexes, the $\operatorname{SCF}(\mathrm{FBXL})$ complexes are intertwined in a complex regulatory network with the APC/C. For example, degradation of FBXL1 is mediated by the APC/C in G1phase [29]. Therefore, SCF(FBXL1) becomes inactivated and is only reactivated when APC/C activity decreases and FBXL1 is stabilized.

The third subfamily of F-box proteins is FBXO, or F-box only. Unlike FBXW proteins, which contain only C-terminal WD40 (structural motif terminating in W-D dipeptide) motifs, and FBXL proteins, which contain only C-terminal LRR (leucine-rich repeat) motifs, FBXO proteins can possess any of 21 other 
homology domains for substrate recognition. There are at least 36 FBXO proteins, many of which are not well understood. However, what is known about FBXO proteins demonstrates their importance in cell cycle regulation (Table 1) [37]. FBXO1, or Cyclin F, contains an amino acid sequence similar to that of Cyclin A and its protein levels oscillate throughout the cell cycle, increasing in S, peaking in $\mathrm{G} 2$, and disappearing in mitosis $[68,69]$. FBXO1 has been reported to bind Cyclin B and facilitate its nuclear import, implicating it in regulation of mitotic entry [70]. The relationship between SCF complexes and the APC/C is further complicated by the fact that FBXO5 (also called EMI1, see above) is an APC/C inhibitor and is itself targeted for degradation by a separate SCF complex containing FBXW1 $[37,41]$. FBXO5, through regulation of APC/C activity, helps to control mitosis by regulating levels of Cyclin A, Cyclin B, securin, and other mitotic targets of the APC/C. Other notable targets of FBXO proteins include TP53, MDM2 (mouse double minute 2), BRCA1 (breast cancer type 1 susceptibility protein), Cyclin D, CHK1 (checkpoint kinase-1), and CDT1 (chromatin licensing and DNA replication factor 1) [37].

The APC/C controls the cell cycle in mitosis and G1-phase and is the counterpart to SCF complexes, which are largely responsible for regulation of Sand G2-phase progression. The APC/C is a large multi-subunit E3 ubiquitin ligase that is essential for proliferation and is the master regulator of mitosis [4, $24,48,52,53]$. It also plays a role in promoting the $\mathrm{G} 1$ progression $[2,27,71$ 74]. An atomic structure of the full complex was determined in 2015, providing insights into the structural and functional roles of each subunit [75]. The APC/C 
has at least 14 subunits that assemble into a $1.5 \mathrm{MDa}$ complex [40]. Within the complex, individual subunits may be grouped into 3 sub-complexes: scaffolding, catalytic and substrate recognition, and tetratricopeptide repeat (TPR) arm subcomplexes $[40,53]$. The catalytic sub-complex contains ANAPC 10 and, like SCF complexes, has a cullin-like subunit, ANAPC2, and a RING-finger subunit, ANAPC11. The scaffolding sub-complex is made up of ANAPC1, ANAPC4, and ANAPC5. The TPR arm, which provides binding sites for scaffolding subunits and coactivators, consists of ANAPC3, ANAPC6, and ANAPC8. In contrast to the approximately 70 F-box proteins that determine substrates for SCF complexes, APC/C substrate selection is determined primarily by association with one of two coactivators: CDC20 and CDH1 [25, 76, 77].

In mitosis, numerous steps are required for full APC/C activation including degradation of inhibitors, association with coactivators, and phosphorylation of core subunits [4]. In G2 and early mitosis, the APC/C binds CDC20 (APC/C(CDC20)), which functions as its substrate selectivity subunit (Table 2) $[24,78]$. CDC20 contains a WD40 repeat domain, which is responsible for binding substrate D-box destruction motifs (RXXLXXXXN) [24]. As previously mentioned, APC/C(CDC20) is inhibited by EMI1 (FBXO5), which prevents substrate recognition by $\mathrm{CDC} 20$ and allows mitotic cyclins to accumulate, driving CDK1 activity and mitotic progression [37, 41, 79]. EMI1 is subsequently phosphorylated in early mitosis by CDK1-Cyclin B and polo-like kinases (PLKs) targeting it for SCF(FBXW1)-mediated ubiquitinylation [80, 81]. Degradation of EMI1, however, is not sufficient to activate APC/C(CDC20). To activate 
Table 2. List of reported $A P C / C(C D C 20)$ and $A P C / C(C D H 1)$ targets

\begin{tabular}{c|c|c} 
Coactivator & CDC20 & CDH1 \\
\hline \multirow{7}{*}{ Target protein } & Cyclin B & Cyclin B \\
Cyclin A & Cyclin A \\
Securin & CDC25A \\
CDKN1B & SKP2 \\
PLK1 & UBE2C \\
Aurora A & FOXM1 \\
Aurora B & Geminin \\
Anillin & CDH1 \\
Geminin & \\
CDC20 & \\
NEK2A &
\end{tabular}


APC/C(CDC20) fully, the mitotic checkpoint, or spindle-assembly checkpoint must be satisfied $[28,82,83]$. The SAC functions to prevent unequal segregation of genetic material between two daughter cells by inhibiting APC/C $(C D C 20)$ until the cell has achieved proper attachment of mitotic spindle microtubules to all sister chromatids [84, 85]. Briefly, the SAC possesses a sensory mechanism that detects unattached kinetochores, promoting formation of a multisubunit mitotic checkpoint complex (MCC) that halts mitotic progression by inhibiting $\mathrm{APC} / \mathrm{C}(\mathrm{CDC} 20)$. The interplay between the SAC and the APC/C is complex will be described in the following paragraphs. Once the SAC is satisfied, the MCC disassembles and the APC/C is phosphorylated at no fewer than 34 mitosisspecific sites [86]. Kraft et. al found that of these 34 sites, 15 can be phosphorylated by CDK1 and 3 by Plk1, but full activation requires phosphorylation only at CDK1 sites [86]. Fully activated APC/C(CDC20), in a negative feedback loop, targets Cyclin B for degradation, leading to CDK1 inactivation and promoting mitotic exit [47, 48, 50, 51]. Concurrently, $\mathrm{APC} / \mathrm{C}(\mathrm{CDC} 20)$ also targets securin, an inhibitor of the cysteine protease separase [49]. APC/C(CDC20)-mediated degradation of securin activates separase, which then cleaves cohesion subunit RAD21 (double-strand-break repair protein rad21 homolog), allowing separation of sister chromatids and promoting anaphase progression [87].

Prior to APC/C activation, $\mathrm{CDH} 1$, the second $\mathrm{APC} / \mathrm{C}$ coactivator, is maintained in a phosphorylated form by CDK1, preventing its association with the APC/C. Following APC/C(CDC20)-mediated Cyclin B degradation and CDK1 
inactivation, $\mathrm{CDH} 1$ is dephosphorylated, allowing its association with the APC/C $(\mathrm{APC} / \mathrm{C}(\mathrm{CDH} 1))[27,88,89] . \mathrm{CDC} 20$ is a substrate of $\mathrm{APC} / \mathrm{C}(\mathrm{CDH} 1)$, and its degradation allows $\mathrm{CDH} 1$ to take over as the substrate specificity subunit (Table 2) $[27,74,78]$. Additionally, as previously mentioned, APC/C(CDC20) relies on phosphorylation to become active, whereas phosphorylation inhibits binding of $\mathrm{CDH} 1$. The opposite regulation of $\mathrm{CDC} 20$ and $\mathrm{CDH} 1$ by phosphorylation is a major factor that allows for the quick exchange of the two coactivators. CDH1, like CDC20, contains WD40 repeats that recognize substrate D-box destruction motifs, but it can also recognize KEN-box motifs (KENXXXN) [25]. In late mitosis, $\mathrm{APC} / \mathrm{C}(\mathrm{CDH} 1)$ continues to target Cyclin B degradation, keeping CDK1 activity low [90]. Many critical regulators of cytokinesis, centrosome replication, and mitotic spindle movement have been documented as APC/C(CDH1) targets [52]. In anaphase, PLK1 is targeted for destruction by APC/C(CDH1), allowing dephosphorylation of proteins involved in elongation of the mitotic spindle [91]. During mitotic exit, $\mathrm{APC} / \mathrm{C}(\mathrm{CDH} 1)$ substrates include both Aurora $\mathrm{A}$ and Aurora $\mathrm{B}$ mitotic kinases, Kinesin-like protein KIFC1 (kinesin family member C1), and the actin-binding protein anillin [92-96]. By promoting the degradation of these late mitotic targets, APC/C $(C D H 1)$ ensures disassembly of the components of the kinetochore, mitotic spindle, and the cytokinetic furrow before the next cell cycle begins [97].

In $\mathrm{G} 1$, the APC/C plays a critical role in preventing premature S-phase entry until pre-replication complexes (pre-RCs) have been assembled at the replication origins. To this end, CDK activity is kept low by continued 
APC/C(CDH1)-mediated cyclin degradation. Additionally, APC/C(CDH1) controls CDK activity by targeting FBXL1 (SKP2), an SCF component with several CDKN targets $[29,42,63,65,66]$. Assembly of the pre-RCs is controlled by $\mathrm{APC} / \mathrm{C}(\mathrm{CDH} 1)$ by targeting geminin, an inhibitor of pre-RC formation, for degradation $[2,73,90]$. When $A P C / C(C D H 1)$ is inactivated in late $\mathrm{G} 1$, geminin is stabilized, which ensures against origin re-licensing until the next cell cycle.

The APC/C is highly regulated, both temporally and in terms of substrate targeting. As has been discussed, this regulation is achieved through its association with adaptors, like $\mathrm{CDC} 20$ and $\mathrm{CDH} 1$, binding of $\mathrm{APC} / \mathrm{C}$ inhibitors, like EMI1, and phosphorylation of the APC/C core. Additionally, in mitosis, the $\mathrm{APC} / \mathrm{C}$ is the target of the SAC $[4,22,28,52,98,99]$.

\section{The APC/C and the SAC}

Equal division of sister chromatids between two daughter cells is an inherently dangerous process, as mistakes result in daughter cells with chromosomal abnormalities, leading to cell death or cancer. For this reason, mitosis is a highly regulated and complex sequence of events. Mitosis is divided into four distinct phases: prophase, metaphase, anaphase, and telophase.

During prophase, chromosomes condense, the nuclear envelope disappears, and the mitotic spindle begins to form (Figure 2). The mitotic spindle consists of microtubules attached to a microtubule organizing complex called the centrosome. Each pair of sister chromatids is joined together at the centromere by a protein complex called the kinetochore. During metaphase, microtubule spindle fibers originating from the two polar centrosomes will attach to the 


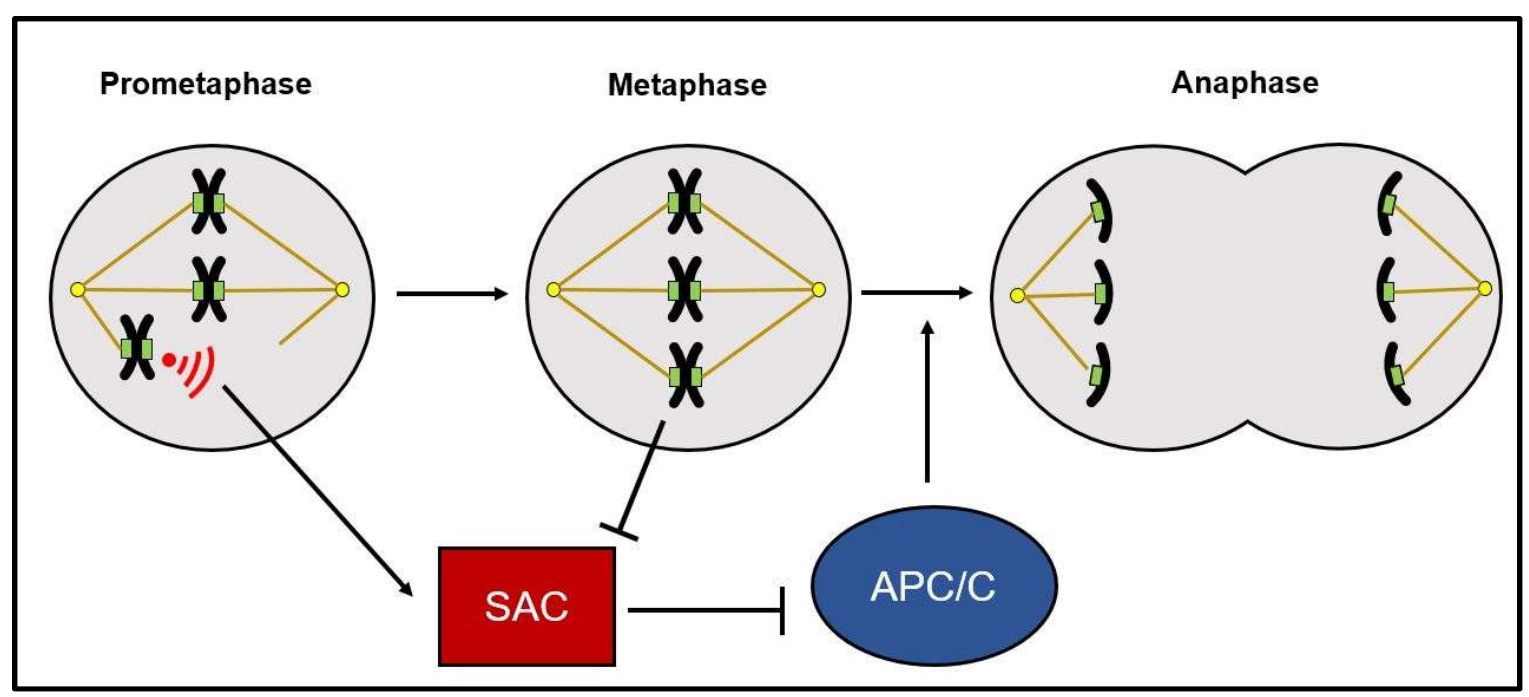

Figure 2. Illustration of the mechanism of the SAC. Unattached kinetochores activate the SAC and produce an inhibitory signal targeting the APC/C. Proper kinetochore attachment, in metaphase, inactivates the SAC, relieving inhibition of the APC/C. Active APC/C promotes the metaphase-anaphase transition. 
kinetochore of each pair of sister chromatids (Figure 2). Microtubule motor proteins located near each of the centrosomes pull the microtubules in opposite directions, placing tension on kinetochores. Because the sister chromatids are still joined at the kinetochore, sister chromatids arrange themselves in the middle of the cell, forming a line called the metaphase plate. In anaphase, cleavage of cohesins, proteins that hold sister chromatids together, allows each sister chromatid to be pulled to opposite ends of the cell by shortening of kinetochoreassociated microtubules (Figure 2). However, before proceeding to anaphase, the cell must pass the spindle assembly checkpoint (Figure 2). Microtubuletargeting drugs, such as paclitaxel, function by exploiting the SAC. By interfering with microtubule function, paclitaxel activates the SAC, leading to APC/C inhibition, mitotic arrest, and apoptosis in cancer cells. In the absence of spindle disruption, the SAC will be satisfied and the cell will progress through anaphase to telophase, during which, sister chromatids reach the poles of the cell, nuclear envelopes begin to form, and spindle microtubules depolymerize. Following telophase is cytokinesis, where the cytosol and its contents are divided into two daughter cells, each with a full set of genetic material.

The SAC is a quality control mechanism that prevents unequal segregation of chromatids between two daughter cells. In metaphase, chromosomes that are unattached or improperly attached to the mitotic spindle promote SAC activation and halt cell division until the mistake can be corrected. Even a single unattached kinetochore is sufficient to activate the SAC and prevent mitotic exit. One of the first studies to point out this phenomenon was 
performed by Raymond Zirkle, who noted that metaphase cells will "wait" when just one chromosome was retarded [100]. Later studies using microtubule depolymerizing agents, such as nocodazole and colchicine, found that the metaphase-anaphase transition was delayed in response to inhibition of mitotic spindle assembly [101]. Fittingly, the newly discovered mitotic checkpoint was designated the spindle-assembly checkpoint. However, the source of the "wait" signal was still unclear. To this end, Rieder et. al showed that mitotic exit could be accelerated by laser ablation of the last unattached kinetochore, providing evidence that the "wait" signal was produced at kinetochores [84]. In the decades following these studies, the mechanisms by which the SAC senses unattached kinetochores and how it mediates mitotic arrest have become clearer, but many unanswered questions remain.

For the SAC to function, the cell must first be able to detect the status of kinetochore attachment and then respond properly. The detection function is performed by a sensory system that monitors kinetochore-spindle attachment. In response to unattached kinetochores, the sensory system will activate an effector system that induces mitotic arrest via APC/C inhibition [85]. The sensory function of the SAC begins at kinetochores, multi-subunit complexes that are the interface between chromosomes and spindle microtubules [85]. The 'outer' layer contains an intricate network of three separate complexes: The KNL1 (kinetochore scaffold 1) complex, the MIS12 (protein MIS12 homolog) complex, and the NDC80 (Kinetochore protein NDC80 Homolog) complex (KMN network). The KMN network promotes spindle attachment by interaction with a calponin- 
homology domain on the NDC80 complex, while the 'inner' layer binds to centromeric chromatin through a histone $\mathrm{H} 3$ variant, CENP-A (histone H3-like centromeric protein A) [102]. Prior to kinetochore-microtubule attachment, all SAC proteins are recruited to kinetochores and, as spindle attachment progresses, levels of these proteins decrease [103]. Some evidence suggests that kinetochores serve as platforms for assembly of the SAC effector, the mitotic checkpoint complex (MCC), but it is still not clear whether full or partial assembly of the MCC occurs here. In either case, it is thought that the complete MCC must be able to diffuse freely throughout the cell to inhibit the APC/C sufficiently [84, 85, 103]. Interestingly, it has been found that the SAC does not work like a switch, but is able to be activated to different levels [104]. Furthermore, the same study found that different microtubule-targeting agents could differentially activate the SAC, causing gradations of APC/C inhibition and varying the extent of mitotic delay.

Aurora Kinase $\mathrm{B}$ is a serine/threonine kinase that is particularly important to SAC function [59]. During mitosis, it is found in high amounts at kinetochores [105]. There, it phosphorylates kinetochore proteins until kinetochore-microtubule attachment occurs, at which point its activity promptly declines. When active, aurora kinase B phosphorylates proteins on both the inner and outer kinetochore, including CENP-A and components of the KMN network. A key signaling function of aurora kinase B is recruitment of MPS1 (monopolar spindle 1 kinase) to kinetochores. MPS1, another kinase and critical SAC signaling molecule, phosphorylates KNL1 at several MELT sequences (Met-Glu-Leu-Thr), creating 
docking sites for additional SAC proteins. These proteins include BUB1 (budding uninhibited by benzimidazoles 1), BUB3 (budding uninhibited by benzimidazoles 3), BUBR1 (budding uninhibited by benzimidazoles 1B; BUB1B), MAD1 (mitotic arrest-deficient 1), MAD2 (mitotic arrest-deficient 2), and CDC20, which are either components of the MCC or promote assembly of the MCC $[59,85,105]$.

The MCC is composed of three SAC proteins MAD2, BUBR1, and BUB3 in addition to CDC20. Incomplete kinetochore-microtubule attachment promotes the assembly of this complex and its inhibition of the APC/C through formation of APC/C ${ }^{\text {Cdc20-MCC }}$ complexes, while proper chromosome biorientation promotes MCC disassembly and APC/C activation. The interplay between the mitotic spindle, the SAC, mitotic progression and the APC/C is exceedingly complex, but an entire class of drugs exploits these connections, providing therapy for numerous diseases.

\section{Importance of this study}

Mitotic inhibitors are drugs that inhibit the cell cycle in mitosis. There are several classes of antimitotics and they are used in the treatment of multiple diseases, including viral skin infections, fungal infections, gout, and multiple types of cancer. Classes of antimitotic drugs include the taxanes (e.g. paclitaxel, docetaxel), vinca alkaloids (e.g. vincristine, vinblastine), colchicine, podophyllotoxin, and griseofulvin. Importantly, these drugs all target a single protein: tubulin. Through their interaction with tubulin, current antimitotics disrupt microtubule dynamics through either stabilization or destabilization of microtubules. In mitosis, the disruption of microtubule dynamics prevents proper 
attachment of kinetochores to mitotic spindle microtubules, leading to SAC activation, APC/C inhibition, and mitotic arrest.

The taxanes are a major class of antimitotics and are commonly used to treat lung, ovarian, and breast cancer. Despite their widespread use, there are numerous issues concerning these drugs. As with most chemotherapeutic agents, the taxanes induce cell death in dividing tissues, not limited to malignant tissues. Additionally, taxanes exhibit dose-limiting neurotoxicity due to their interference with neuronal microtubule dynamics. A major issue that continues to receive significant attention is drug resistant cancers. Tumors frequently become resistant to current antimitotics, including the taxanes, by mutations in tubulin or SAC proteins. Thus, since all current antimitotics target tubulin, cancers with these mutations are resistant to the entire class of drugs. Development of drugs that target the regulators of mitosis, such as components of the UPS, will provide significant benefits over the current antimitotic drugs.

There are several examples of drugs that target members of the UPS for cancer chemotherapy, including the general proteasome inhibitor, bortezomib, for treatment of multiple myeloma and mantle cell lymphoma. While bortezomib has a measurable therapeutic index, it inhibits all protein degradation, which can cause unwanted cytotoxic effects [34]. In an attempt to reduce the off-target effects caused by overall inhibition of protein degradation, some groups have developed small molecules to inhibit individual E3 ubiquitin ligases. One example is Pevonedistat (MLN4924), an inhibitor of NEDD8-activating enzyme [106]. The activity of SCF complexes requires neddylation (addition of a small, ubiquitin-like 
protein) of cullin subunits. Pevonedistat, by blocking SCF neddylation, serves as an SCF inhibitor. Despite increased specificity over bortezomib, phase 1 clinical trials with pevonedistat reported only modest activity [107]. Through inhibition of all SCF ligases, pevonedistat blocks proteasomal degradation of $\sim 20 \%$ of proteins. Taking into account the breadth of SCF ubiquitin ligase activity, total inhibition of SCF ligases is likely an ineffective approach. Additionally, SCF complexes display overlapping substrate specificity due to the nature of targeting by F-box proteins. As mentioned previously, the redundancy of F-box substratetargeting highlights the impracticality of SCF inhibition for achieving specific therapeutic effects.

This thesis characterizes a new class of antimitotic chemotherapeutics targeting the major regulator of cell cycle progression, the anaphase promoting complex/cyclosome (APC/C). The strategy differs from traditional approaches, in that core structures necessary for intermolecular interactions are targeted.

As previously mentioned, the APC/C catalytic core contains a cullin (ANAPC2) and a zinc RING finger (ANAPC11) subunit [5]. At the inception of this project, there were no published structures of the APC/C or its subunits. However, crystal structures of proteins homologous to ANAPC11 and ANAPC2 were available. PDB files 1LDJ for CUL1 (ANAPC2 homologous) and RBX1 (APC11 homologous) were obtained from the protein database maintained by the National Library of Medicine. ClustalW software was used to create alignment files, and homology models for ANAPC2 and ANAPC11 were generated with Modeller 9v1 [108]. ANAPC2 and ANAPC11 were joined by insightll software by 
aligning ANAPC11 to RBX1 and ANAPC2 to CUL1. Images of the generated homology models were exported from either insightll or Chimera software. ANAPC2:ANAPC11 homology models were then used to for in silico docking to identify compounds predicted to bind the ANAPC11-binding region of ANAPC2 (Figure 3A). More recently, the full APC/C structure was determined by cryoelectron microscopy and added to the protein database. The homology models were compared to the published structures for ANAPC2-CTD and ANAPC11 (protein database file 4UI9) and found to be highly similar (Figure 3B). Two sites within the ANAPC11-binding region of ANAPC2 were screened using SurflexDock and the ZINC drug-like library, which contained, at the time of screening, over 3 million compounds. The results were scored and the top $\sim 50$ candidate compounds were selected for each site. A total of nine compounds, four targeting one site and five targeting the other, were ordered for studies. From these nine compounds, preliminary data indicate that four are cytotoxic in various cancer cell lines. These four hit compounds include three compounds classified as amino-2-propanols and one as a triazospiro compound (Figure 3CF). The predicted ANAPC2 binding regions for the the amino-2-propanols and triazospiro compounds are distinct but overlapping (Figure 3A, turquoise and green). 


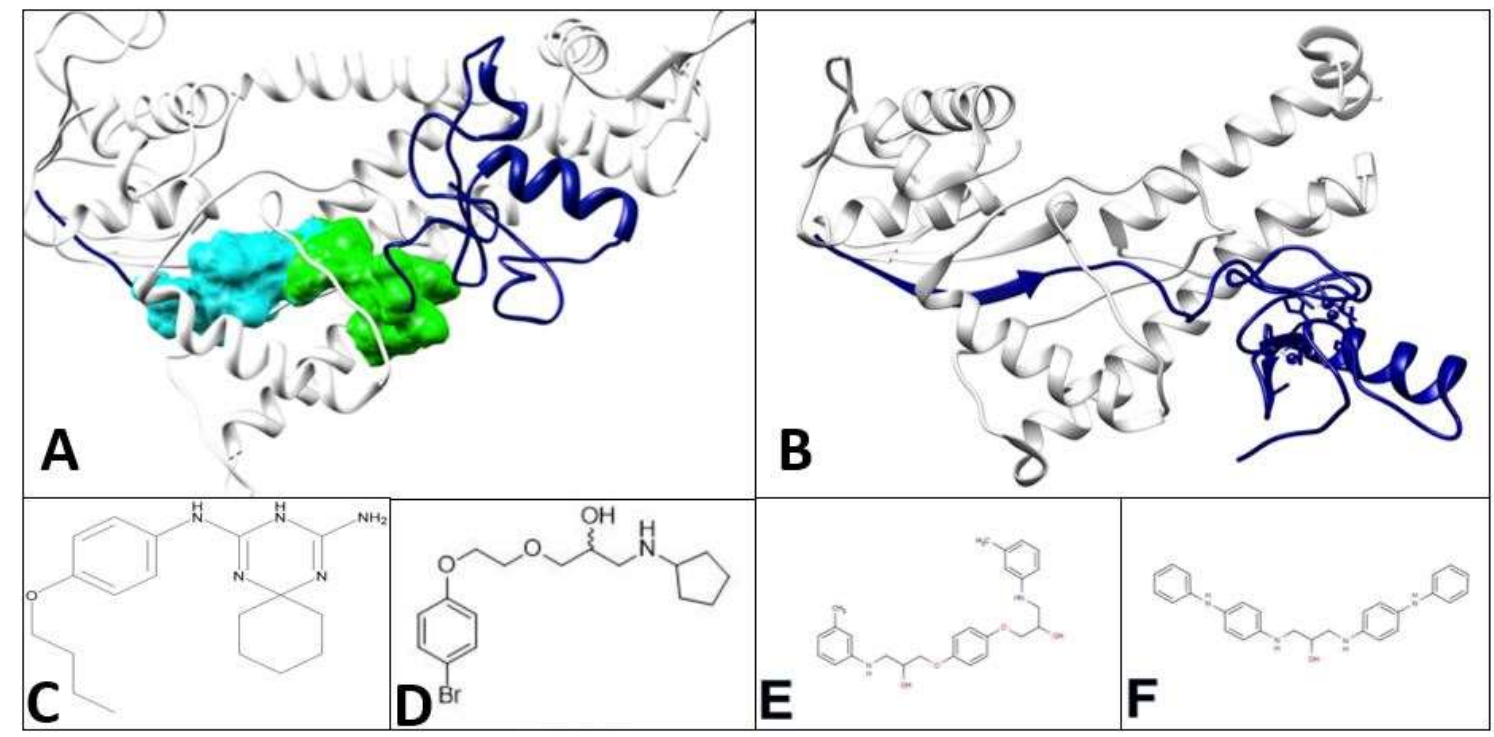

Figure 3. Models of the ANAPC2:ANAPC11 binding interaction and structures of predicted inhibitors. A) Homology models of the C-terminal domain (CTD) of ANAPC2 (white) binding ANAPC11 (blue). Regions targeted in silico are represented in turquoise and green. B) Published cryo-electron microscopy structures of the binding interaction of ANAPC2-CTD (white) and ANAPC11 (blue). The lower panels show the structures of four predicted inhibitors: C) Compound 3; D) Compound 8; E) Compound 10; F) Compound 11. Structures in panel A courtesy of B.F. Taylor and J.O. Trent. Panel B structures from PDB file 4UI9. 
Based on the role of the $\mathrm{APC} / \mathrm{C}$ in cell cycle regulation, the overarching hypothesis is that compounds predicted to inhibit the APC/C will induce mitotic arrest and cell death in malignant cells. In this thesis, the following are demonstrated: 1) Hit compounds are cytotoxic in multiple cancer types, including lung, ovarian, pancreatic, and melanoma cancers; 2) Hit compounds induce cell cycle mitotic or G1-arrest depending on the functionality of the SAC; 3) Hit compounds induce apoptosis selectively in malignant cells. This thesis, and future work, will provide the groundwork for development of these compounds into a novel class of antimitotic chemotherapeutics, which are critically needed for treatment of anti-microtubule-resistant tumors. Additionally, these compounds will serve as useful tools for studying the mechanisms of mitosis and the APC/C. 


\section{METHODS AND MATERIALS}

\section{CELL CULTURE}

Cells were cultured at $37^{\circ} \mathrm{C}$ with $5 \% \mathrm{CO}_{2}$ in a humidified incubator. A549 and A375 cells were cultured in Dulbecco's Modified Eagle's Medium (Hyclone, Logan, UT) supplemented with $10 \%$ fetal bovine serum (Hyclone), $100 \mathrm{U} / \mathrm{mL}$ penicillin and $10 \mathrm{U} / \mathrm{mL}$ streptomycin. A2780/CP70 and H460 cells were cultured in RPMI-1640 (Hyclone) supplemented with 10\% fetal bovine serum (Hyclone), $100 \mathrm{U} / \mathrm{mL}$ penicillin and $10 \mathrm{U} / \mathrm{mL}$ streptomycin. SKOV-3 cells were cultured in McCoy's 5A (Hyclone) supplemented with 10\% fetal bovine serum (Hyclone), $100 \mathrm{U} / \mathrm{mL}$ penicillin and $10 \mathrm{U} / \mathrm{mL}$ streptomycin. Telomerase-immortalized diploid fibroblasts, tGM24 cells, were cultured in Eagle's MEM containing Earle's salts (GIBCO-BRL) supplemented with $0.1 \mathrm{mM}$ non-essential amino acids, $2 \mathrm{mM}$ Lglutamine $10 \%$ fetal bovine serum (Hyclone), $100 \mathrm{U} / \mathrm{mL}$ penicillin and $10 \mathrm{U} / \mathrm{mL}$ streptomycin. SK-Mel-28 cells were cultured in a-MEM (Hyclone) supplemented with $10 \%$ fetal bovine serum (Hyclone), $100 \mathrm{U} / \mathrm{mL}$ penicillin and $10 \mathrm{U} / \mathrm{mL}$ streptomycin. HBEC3-KT cells were cultured in Keratinocyte-SFM (GIBCO) supplemented with $50 \mu \mathrm{g} / \mathrm{mL}$ bovine pituitary extract, $5 \mathrm{ng} / \mathrm{mL}$ recombinant human EGF, and $100 \mathrm{U} / \mathrm{mL}$ penicillin and $10 \mathrm{U} / \mathrm{mL}$ streptomycin. 


\section{ALAMARBLUE ASSAYS}

Cells were seeded in 96-well plates at 8000 cells/ well (A549, H460) or 2000 cells/ well (HBEC3-KT) and allowed to attach overnight. Cells were treated with seven concentrations of compounds $3(5-60 \mu \mathrm{M}), 8(10-100 \mu \mathrm{M}), 10(5-60$ $\mu \mathrm{M})$, or $11(1-20 \mu \mathrm{M})$. DMSO (0.1\%), paclitaxel (100 nM), and staurosporine (10 $\mu \mathrm{M})$ were used as controls. Concentration ranges and treatment times for lead compounds were chosen based on prior photomicroscopy studies of cell morphology and cell death. Cells were incubated with compounds for $48 \mathrm{~h}$, at which point alamarBlue (Bio-Rad, Hercules, CA) was added to each well to $10 \%$ total volume. Plates were incubated with alamarBlue for $4 \mathrm{~h}$ at $37^{\circ} \mathrm{C}$ and $5 \%$ $\mathrm{CO}_{2}$. The fluorescence of each well was measured with an excitation wavelength of $530 \mathrm{~nm}$ and an emission wavelength of $590 \mathrm{~nm}$. 


\section{COLONY FORMING ASSAYS}

Cells were plated in triplicate 6-well plates at a 100 cells/well (3 wells) or 500 cells/well ( 3 wells) and allowed to attach overnight. Cells were then exposed to DMSO or compounds at the indicated concentrations dissolved in DMSO (final [DMSO] $=0.1 \%)$. The time of incubation with compounds varied from 5 to 14 days between cell lines due to differences in doubling times. Generally, colonies become visible when clusters reach 32 cells or more, so $\geq 5$ doublings are required to fit the definition of a colony. Following incubation with compounds, colonies were fixed and stained with crystal violet. Stained colonies were counted and the colony forming efficiency (CFE) was calculated by dividing the number of colonies formed by the number of cells plated. These values are then used to calculate the colony forming efficiency relative to the DMSO control by the following formula:

$$
\text { Colonies }(\% \text { control })=\frac{\text { Compound CFE }}{\text { DMSO CFE }} * 100
$$




\section{MITOTIC INDEX ANALYSIS}

Cells were seeded in 6-well plates at $1.5 \times 10^{5}$ cells/ well. After allowing cells to attach for $24 \mathrm{~h}, \mathrm{DMSO}$, paclitaxel (100 nM), or compounds 3, 8,10 , and 11 were added directly to the media. Concentration ranges and treatment times for lead compounds were chosen based on prior photomicroscopy studies of cell morphology and cell death. After $24 \mathrm{~h}$ cells were harvested for mitotic index determination by trypsinization and combined with media and wash. Cells were collected by centrifugation and pellets were resuspended and swollen in $2.5 \mathrm{~mL}$ hypotonic solution $(0.075 \mathrm{M} \mathrm{KCl})$. The suspension was incubated at room temperature for $15 \mathrm{~min}$. Next, $0.25 \mathrm{~mL}$ fixative solution (3:1 methanol: acetic acid) was added, and cells were pelleted by centrifugation. Pellets were resuspended in $2.5 \mathrm{~mL}$ fixative solution and incubated at room temperature for at least $20 \mathrm{~min}$. Cells were pelleted again and resuspended in $2.5 \mathrm{~mL}$ fresh fixative solution. Aliquots were then dropped onto glass slides, air dried, and stained with Wright Giemsa solution. Slides were examined under a microscope for determination of mitotic index, mitotic catastrophe, and apoptosis. A minimum of three slides per treatment were prepared and 200 cells were counted per slide. 


\section{G1/S SYNCHRONIZATION}

Cells were synchronized at G1/S by double thymidine block. Experiments were performed with $5 \times 10^{5}$ cells in $6 \mathrm{~cm}$ dishes. Cells were plated and allowed to attach overnight, followed by incubation with $2 \mathrm{mM}$ thymidine for $20 \mathrm{~h}$. Thymidine was removed to allow cells to re-enter the cell cycle by aspirating media, washing twice with PBS, and adding fresh media. After $8 \mathrm{~h}$, cells were treated with $2 \mathrm{mM}$ thymidine for $16 \mathrm{~h}$. Cells were then allowed to re-enter the cell cycle by removing media, washing twice with PBS, and adding fresh media. DMSO $(0.1 \%)$, compounds $3(10 \mu \mathrm{M}), 8(40 \mu \mathrm{M}), 10(10 \mu \mathrm{M})$, and $11(5 \mu \mathrm{M})$, or nocodazole $(100 \mathrm{nM})$ were added directly to the media $5 \mathrm{~h}$ after release from G1/S block. Total cellular lysates were prepared every hour for the next $7 \mathrm{~h}$. 


\section{WESTERN BLOTTING}

Cells were lysed with lysis solution (10 mM Tris- $\mathrm{HCl}$ pH 7.4, 1 mM EDTA, $0.1 \%$ sodium dodecyl sulfate, $1 \mathrm{mM}$ phenylmethylsulphonylfluoride, $1 \mathrm{mM}$ $\mathrm{Na}_{3} \mathrm{VO}_{4}$, and 1X cOmplete ${ }^{\mathrm{TM}}$ Protease Inhibitor Cocktail (Roche, \#04693116001, Basel, Switzerland)). Lysates were sonicated twice for 2-3 s on ice and centrifuged at $13,000 \times \mathrm{g}$ for $15 \mathrm{~min}$ at $4^{\circ} \mathrm{C}$ to remove debris. Total protein concentration in supernatant was determined by BCA assay (Pierce, \#23225, Waltham, MA) using bovine serum albumin as standard. Proteins were loaded (20-30 $\mu \mathrm{g} / \mathrm{lane})$ and resolved by sodium dodecylsulfate-polyacrylamide gel electrophoresis in 12\% Mini-PROTEAN® TGX ${ }^{\mathrm{TM}}$ Precast Protein Gels (Bio-Rad, \#4561046, Hercules, CA). Proteins were transferred to nitrocellulose membranes, which were stained with Ponceau S (Sigma, St. Louis, MO) to check for equal protein loading and transfer. Membranes were blocked in $5 \%$ milk in TBST (10 mM Tris-HCl pH 7.4, $150 \mathrm{mM} \mathrm{NaCl,} \mathrm{0.1 \%} \mathrm{Tween-20)} \mathrm{for} 2 \mathrm{~h}$ at room temperature. Membranes were probed with mouse monoclonal antibodies for $ß$ actin (Sigma, St. Louis, MO, \# A5441, 1:10,000 dilution) and cyclin B (BD Transduction Laboratories, Franklin Lakes, NJ, \#610219, 1:500 dilution) or rabbit monoclonal antibodies for securin (Cell Signaling Technology, Danvers, MA, \#13445, 1:1000 dilution) and vinculin (Cell Signaling Technology, \#13901, 1:1000 dilution). Secondary antibodies (Cell Signaling Technology, Danvers, MA, antimouse IgG, \#7076, 1:3000 dilution or anti-rabbit, \#7074, 1:2000 dilution) conjugated to horseradish peroxidase were bound to primary antibodies and protein bands for $ß$-actin, cyclin B, securin, and vinculin were detected using 
enhanced chemiluminescence (ECL) Plus Western Blotting Substrate (Pierce, \#32123, Waltham, MA). Bands were developed using a ChemiDoc XRS System (Bio-Rad, \#1708265, Hercules, CA). ß-actin and vinculin served as the loading control. 


\section{FLOW CYTOMETRY}

Cells were harvested by trypsinization and fixed in $1 \mathrm{~mL}$ of ice-cold $70 \%$ ethanol overnight at $4^{\circ} \mathrm{C}$. Following fixation, cells were centrifuged at $1,500 \times \mathrm{g}$ and cell pellets were resuspended in $500 \mu \mathrm{L}$ PBS containing $10 \mu \mathrm{g} / \mathrm{mL}$ propidium iodide and $100 \mathrm{U} / \mathrm{mL}$ RNase A (Sigma) for $30 \mathrm{~min}$ at room temperature. Stained cells were analyzed on a FACSCalibur (Becton Dickinson, San Jose CA) using doublet discrimination. Propidium iodide fluorescence was collected on FL2 (585/42 nm) using linear amplification, and at least 20,000 events/ sample were

analyzed. CellQuest software (Beckton Dickinson) was used for data collection and Modfit software (Verity Software House, inc., Topsham ME) was used for analysis of cell cycle distribution. 


\section{APOPTOSIS ASSAYS}

Apoptosis was measured using the Apo-ONE® Homogeneous Caspase3/7 Assay from Promega. This assay utilizes a pro-fluorescent caspase-3/7 substrate that is converted to a highly fluorescent molecule upon cleavage by caspase-3/7. Caspase- $3 / 7$ activity, and therefore apoptosis, is directly correlated to the amount of fluorescence detected. Cells were grown at either 7500 cells/ well (A2780/CP70) or 3000 cells/ well (SKOV-3, tGM24) in 96-well plates in 100 $\mu \mathrm{L}$ media for $24 \mathrm{~h}$. A549, H460, and HBEC3-KT cells were treated with DMSO (0.1\%), compounds $3(5-60 \mu \mathrm{M}), 8(10-80 \mu \mathrm{M}), 10(1-25 \mu \mathrm{M})$, and $11(1-15 \mu \mathrm{M})$. A2780/CP70 cells were treated with DMSO $(0.1 \%)$, compounds $8(20-80 \mu \mathrm{M}), 10$ (1-20 $\mu \mathrm{M})$, and $11(1-20 \mu \mathrm{M})$, or paclitaxel (100 nM). SKOV-3 and tGM24 cells were treated with DMSO $(0.1 \%)$, compounds $8(10-60 \mu \mathrm{M}), 10(1-10 \mu \mathrm{M})$, and 11 (1-10 $\mu \mathrm{M})$, or paclitaxel $(100 \mathrm{nM})$. Cells were incubated for $24 \mathrm{~h}$ (A2780/CP70, SKOV-3), 36 h (A549, H460, HBEC3-KT), or 48 h (tGM24). Concentration ranges and treatment times for lead compounds were chosen based on prior photomicroscopy studies for induction of apoptosis. Cells were exposed to ApoONE® Caspase-3/7 Reagent (Promega, Madison, WI) by adding $100 \mu \mathrm{L}$ to each well. Contents of the wells were gently mixed using a plate shaker at $300-500$ rpm, and cells were incubated for an additional $1 \mathrm{~h}$, prior to fluorescence measurement. The fluorescence of each well was then measured using $499 \mathrm{~nm}$ excitation and 521 emission wavelengths. 


\section{RESULTS}

\section{ALAMARBLUE SCREENING IN MULTIPLE CELL TYPES}

The purpose of this part of the study was to perform initial screening of candidate compounds for cytotoxic properties. Compounds were initially screened by alamarBlue assays. These assays are similar to tetrazolium dyebased MTT (3-(4,5-dimethylthiazol-2-yl)-2,5-diphenyltetrazolium bromide) assays in that they both utilize reporter dyes to measure the reductive capacity, and, therefore, the metabolic activity, of the cell. Additionally, both assays allow rapid screening of multiple compounds. AlamarBlue differs in that it can be added directly to the cell culture media, unlike MTT, which requires washing steps. Inhibition of the APC/C is predicted to cause mitotic arrest, so the wash steps with MTT assays may result in the loss of these loosely-attached cells. Thus, alamarBlue was used because it allows for the measurement of cells in any cell cycle phase. Several predicted APC/C inhibitors $(3,10$, and 11) were found to reduce viability effectively in multiple cell lines representing malignant and nonmalignant lung tissues (Figure 4). While these assays indicate that compounds have no therapeutic window, further investigations are necessary to determine if compounds induce cell death or simply prevent cell division (cytostasis). 

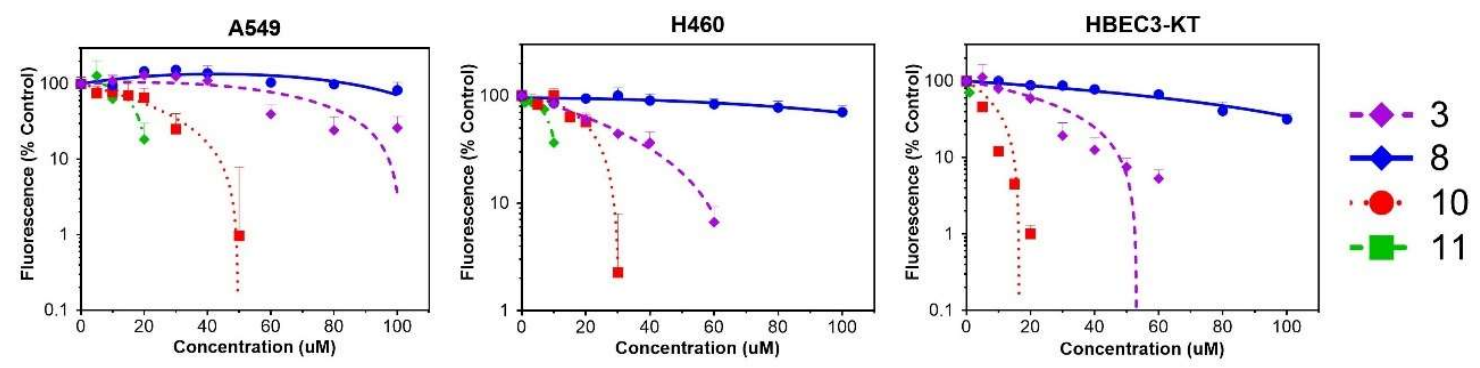

Figure 4. Hit compounds reduce viability in lung cell lines. AlamarBlue assays were performed with compounds 3, 8, 10, and 11 in A549 and H460, malignant lung cell lines, and HBEC3-KT, non-malignant lung cells. Cells were exposed to compounds $3,8,10$, and 11 at the indicated concentrations for $48 \mathrm{~h}$. 


\section{COLONY FORMING ASSAYS IN MULTIPLE CELL TYPES}

AlamarBlue assays don't distinguish between cytostasis and reduced viability. While alamarBlue assays are a useful tool for screening, they are not a true measure of inhibition of replicative ability. To confirm the results of the alamarBlue viability date, colony forming assays, which provide a direct measure of the effect compounds have on replicative capacity, were performed. The results of colony forming assays were consistent with the alamarBlue data, indicating that compounds $3,8,10$, and 11 dose-dependently reduce colony formation (Figure 5). Compounds effectively reduced colony formation in cell lines representing malignant lung, ovarian, pancreatic, and melanoma tissues. Compounds also reduced colony formation in HBEC3-KT, non-malignant lung cells, and tGM24, telomerase-immortalized diploid fibroblasts. These nonmalignant cells, however, were found to be alive and attached to the plate, but had not divided to form colonies (Figure 6). The observation that compounds are cytotoxic to malignant cells, but cytostatic in non-malignant cells provides evidence for a therapeutic window. Interestingly, when compared to DMSOtreated cells, HBEC3-KT cells exposed to hit compounds appear much smaller, suggesting that compounds interfere with pathways regulating cell growth and metabolism. Therefore, it will be necessary to investigate these pathways as sources of off-target effects, in the future. 

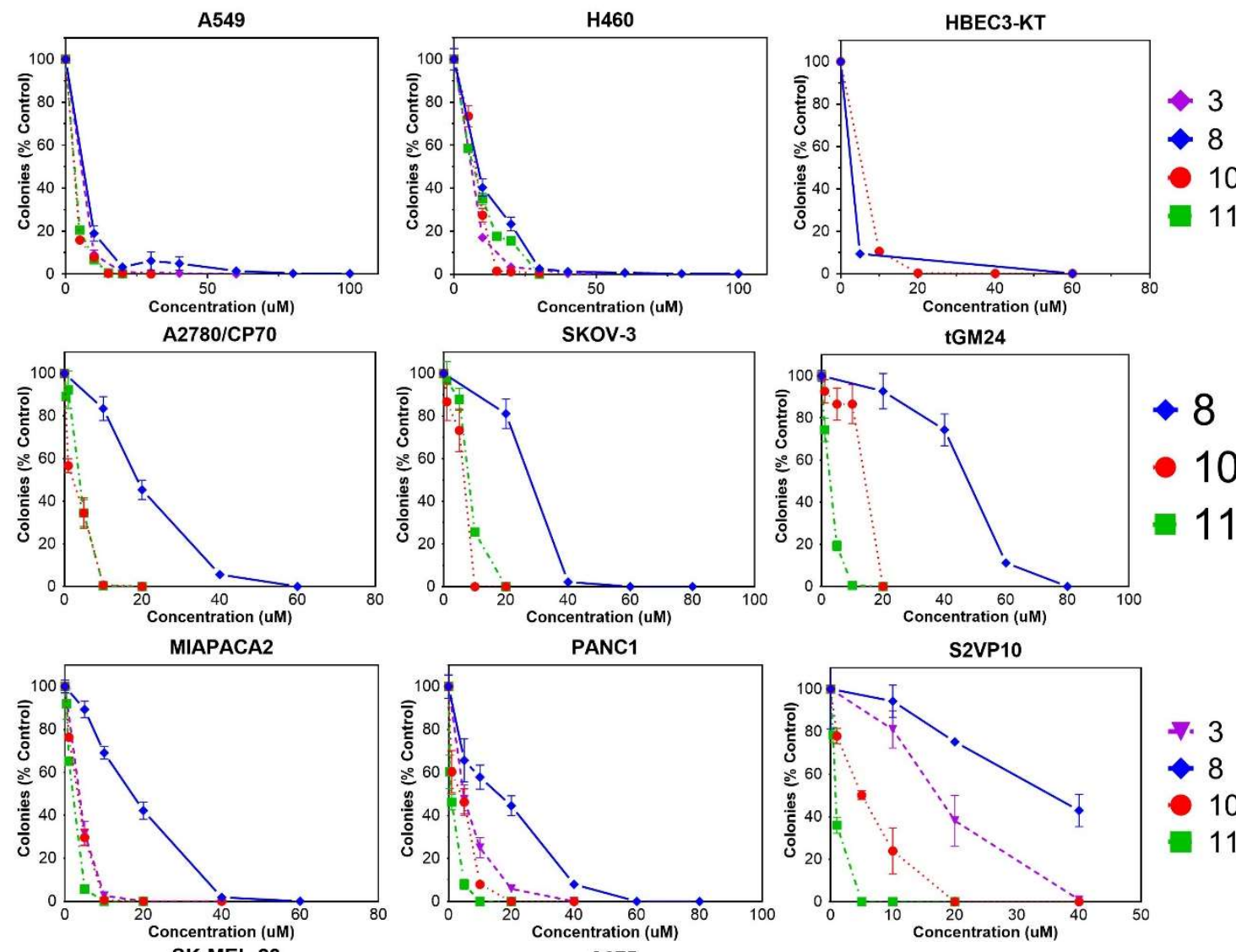

*. 3
7
710
11
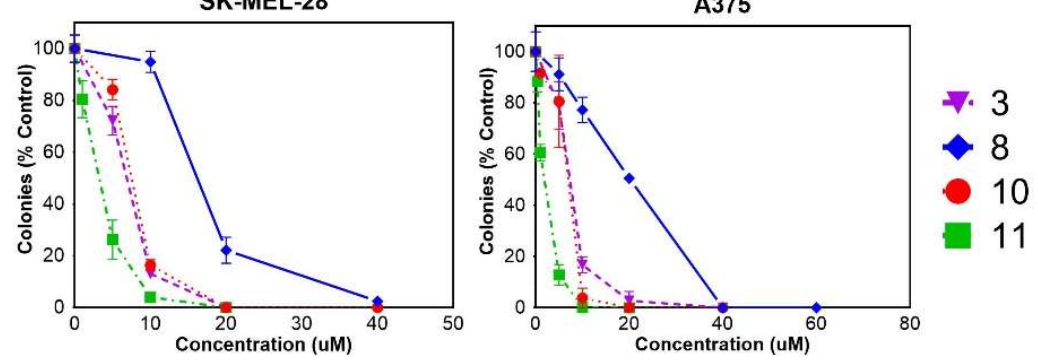

Figure 5. Hit compounds reduce colony forming ability of multiple lung,

ovarian, pancreatic, and melanoma cell lines. Colony forming assays

performed in lung (top row), ovarian and fibroblasts (second row), pancreatic

(third row), and melanoma (fourth row) cell lines. Lung cell lines (A549, H460,

HBEC3-KT) were treated with compounds $3,8,10$, and 11 at the indicated

concentrations. Ovarian cell lines (A2780/CP70, SKOV-3) and fibroblasts

(tGM24) were treated with compounds 8,10 , and 11 at the indicated

concentrations (data courtesy of Doug Saforo). Pancreatic (MIAPACA2, PANC1, 
S2VP10) and melanoma (SK-MEL-28, A375) cell lines were treated with compounds $3,8,10$, and 11 (data courtesy of Brian Sils).

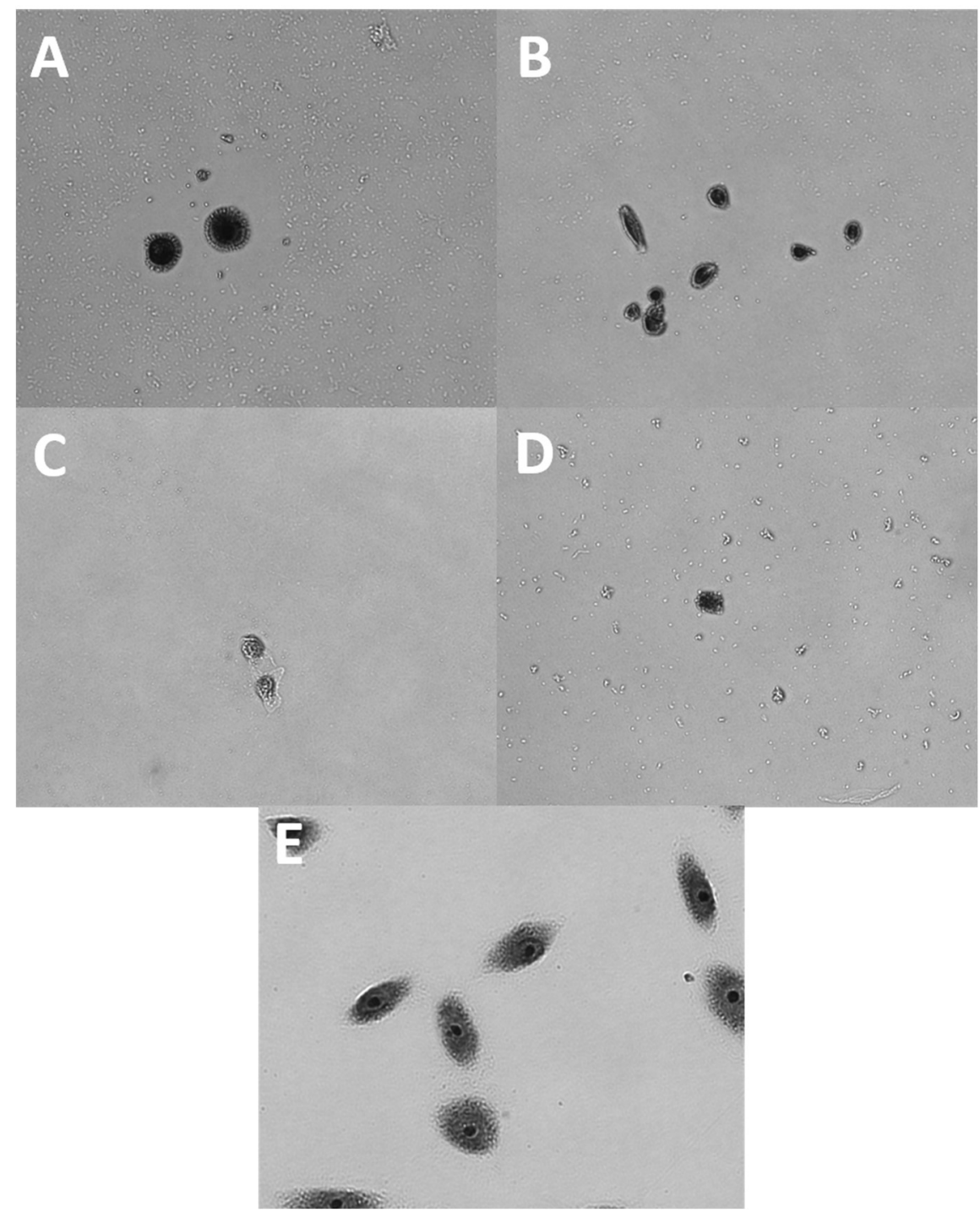

Figure 6. Compounds cause cytostasis in non-malignant lung cells. Phase contrast photomicrographs of HBEC3-KT cells stained with crystal violet following exposure to A) compound $3(20 \mu \mathrm{M}), \mathrm{B})$ compound $8(20 \mu \mathrm{M}), \mathrm{C})$ compound 10 $(20 \mu \mathrm{M}), \mathrm{D})$ compound $11(2 \mu \mathrm{M})$, or E) DMSO. All photos were taken at 10X magnification. 


\section{MITOTIC INDEX ANALYSIS OF MULTIPLE CELL TYPES}

The cytotoxicity of several hit compounds has been demonstrated, but it is possible that this toxicity could be due to off target effects. These compounds are predicted to inhibit the APC/C, which is essential for mitotic progression, particularly the metaphase-anaphase transition. Thus, an increase in the mitotic index in cells exposed to these compounds would be consistent with APC/C inhibition. Therefore, mitotic index analyses were performed to determine the effect of compounds. The results of these experiments indicate that compounds $3,8,10$, and 11 significantly increase mitotic index compared to DMSO controls (Figure 7). Compounds increased mitotic index in multiple cell types, including malignant and non-malignant lung and ovarian cells (Figure 7). However, these results and the results of colony forming assays indicate that non-malignant cells are able to survive mitotic arrest induced by these compounds, while malignant cells undergo cell death. 


\section{Mitotic Index Analysis}
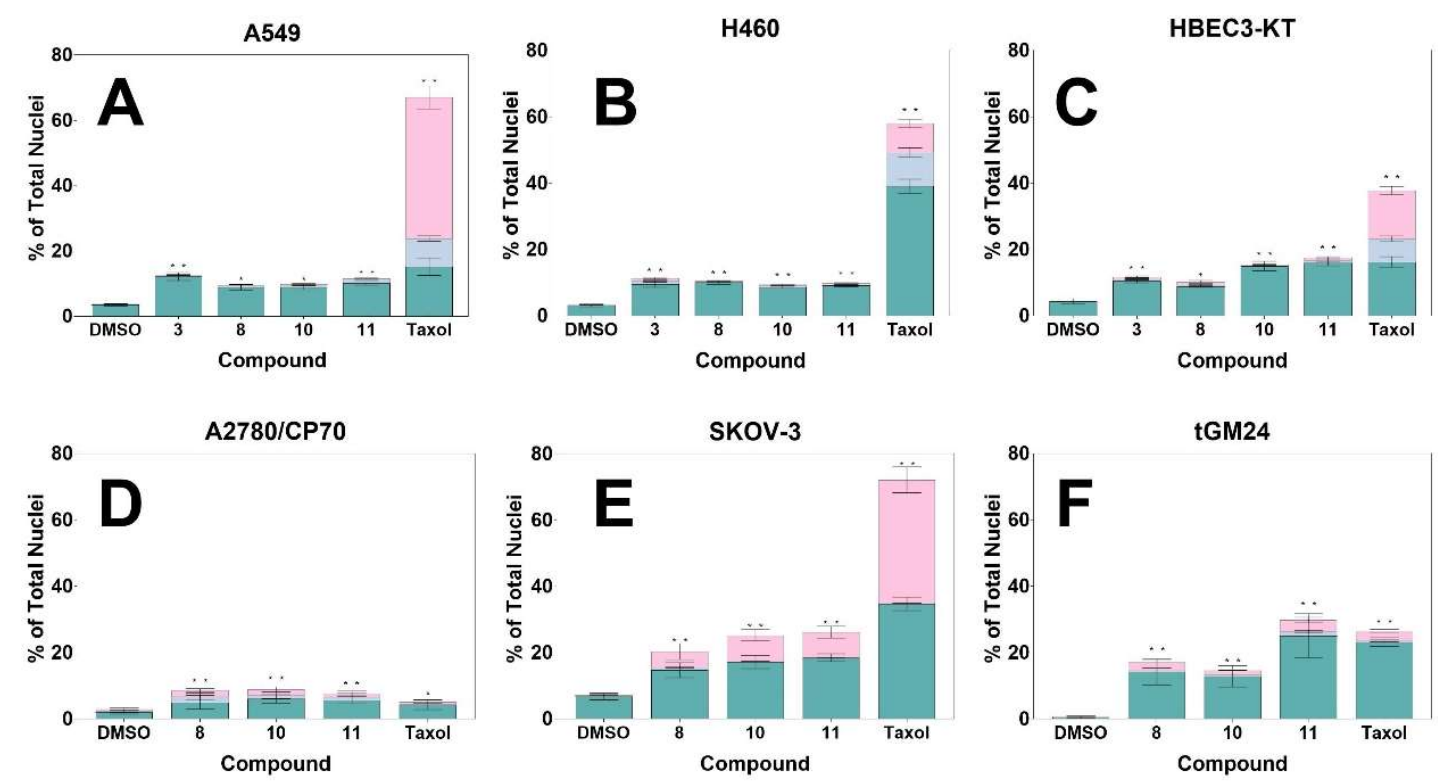

Mitoses

Catastrophes

\section{Apoptoses}

Figure 7. Lung, ovarian, and fibroblast cells exposed to hit compounds have increased populations in mitosis. Mitotic indices determined in lung (AC), ovarian (D, E) and telomerase-immortalized diploid skin fibroblasts $(F)$. Lung cell lines A549 (A), H460 (B), and HBEC3-KT (C) were treated with Taxol (100 $n M)$ and compounds $3(10 \mu \mathrm{M}), 8(60 \mu \mathrm{M}), 10(10 \mu \mathrm{M})$, and $11(5 \mu \mathrm{M})$.

Treatments for A2780/CP70 (D) were Taxol (10 nM) and compounds $8(5 \mu \mathrm{M})$, $10(0.4 \mu \mathrm{M})$, and $11(0.4 \mu \mathrm{M})$. SKOV-3 $(\mathrm{E})$ were treated with Taxol $(50 \mathrm{nM})$ and compounds $8(20 \mu \mathrm{M}), 10(1 \mu \mathrm{M})$, and $11(1 \mu \mathrm{M})$. tGM24 $(\mathrm{F})$ cells were treated with Taxol (50 nM) and compounds $8(20 \mu \mathrm{M}), 10(1 \mu \mathrm{M})$, and $11(1 \mu \mathrm{M})$. Percent difference in mitoses was analyzed by one-way ANOVA $(*, \mathrm{P}<0.05 ; * *$, $P<0.01)$ 


\section{STABILITY OF APC/C SUBSTRATES IN SYNCHRONIZED A375 CELLS}

The APC/C is essential for mitotic progression, particularly the metaphase-anaphase transition. Non-functional APC/C will be unable to target its substrates for ubiquitinylation and degradation. These substrates include cyclin $\mathrm{B}$, whose degradation is required for mitotic exit, and securin, which must be degraded to allow anaphase progression. As a counterpoint, inhibition of the APC/C does not necessarily result in stabilization of its substrates. For example, proteolysis of $A P C / C(C D C 20)$ substrate cyclin $A$ was not delayed in the presence of active SAC in vitro [109]. Additionally, NEK2A (never in mitosis gene a-related kinase 2), has been shown to be targeted by APC/C(CDC20) independent of SAC activation [110]. Furthermore, some cancers with weakened checkpoints may undergo "mitotic slippage," whereby cells slowly degrade cyclin B and prematurely exit mitosis due to incomplete APC/C inhibition [111, 112]. While there is no evidence suggesting that cyclin B or securin can be degraded when the APC/C is completely inhibited, these studies highlight the complex nature of APC/C regulation and activity. Inhibition of the APC/C is most likely to cause cell cycle arrest in mitosis. However, the APC/C also contributes to progression through G1-phase of the cell cycle [2, 27]. Thus, it is possible that APC/C inhibition may result in $\mathrm{G} 1$-arrest, or both G1- and mitotic-arrest.

To provide further evidence that hit compounds target the APC/C and characterize the mechanism of cell cycle arrest, the kinetics of cyclin B and securin degradation in synchronized A375 cells were analyzed. Synchronization of cultures was performed because it is possible that hit compounds targeting the 
APC/C may not inhibit the total APC/C population. Incomplete inhibition of the APC/C would allow slow degradation of its target proteins, resulting in mitotic delay, similar to mitotic slippage by weakened checkpoints, not mitotic arrest. While mitotic progression may be slowed, the population of mitotic cells is likely to be low at any certain time. Therefore, if hit compounds do not completely inhibit APC/C activity, their effects may be masked in asynchronous cultures. To increase the likelihood of obtaining highly synchronous cultures, A375 cells were chosen, because they have a short doubling time. Following G1/S blockade, synchronized cells were allowed to re-enter the cell cycle for $5 \mathrm{~h}$ before adding compounds. After addition of compounds, APC/C targets, securin and cyclin B, were measured every hour for $7 \mathrm{~h}$ by western blot (Figure 8 ). In DMSO-treated cells, cyclin B and securin levels steadily increase from 6-9 $\mathrm{h}$ after release, followed by a sharp decline from $10-12 \mathrm{~h}$, indicating that these cells are exiting mitosis between 9 and $10 \mathrm{~h}$ after G1/S. Nocodazole is commonly used to arrest cells in mitosis during prometaphase. Consistent with mitotic arrest, the levels of cyclin B and securin in nocodazole-treated cells remained elevated through $12 \mathrm{~h}$ after $\mathrm{G} 1 / \mathrm{S}$, our final timepoint. Compounds 3, 8, 10, and 11 had variable effects on cyclin B and securin levels. Similar to nocodazole, compound 3 completely stabilized cyclin B and delayed securin degradation until $12 \mathrm{~h}$ after $\mathrm{G} 1 / \mathrm{S}$, consistent with APC/C inhibition and mitotic arrest. Conversely, compound 8 had no effect on cyclin B levels, when compared to DMSO, but securin was stabilized. Cells treated with compounds 10 and 11 displayed delayed kinetics of degradation for both cyclin B and securin. In these cells, cyclin B and securin 
levels began to decline after $9 \mathrm{~h}$, but the rate of decline was slowed in comparison to DMSO. These delayed kinetics of degradation suggest compounds 10 and 11 cause partial inhibition of the APC/C, leading to mitotic delay, not mitotic arrest. 


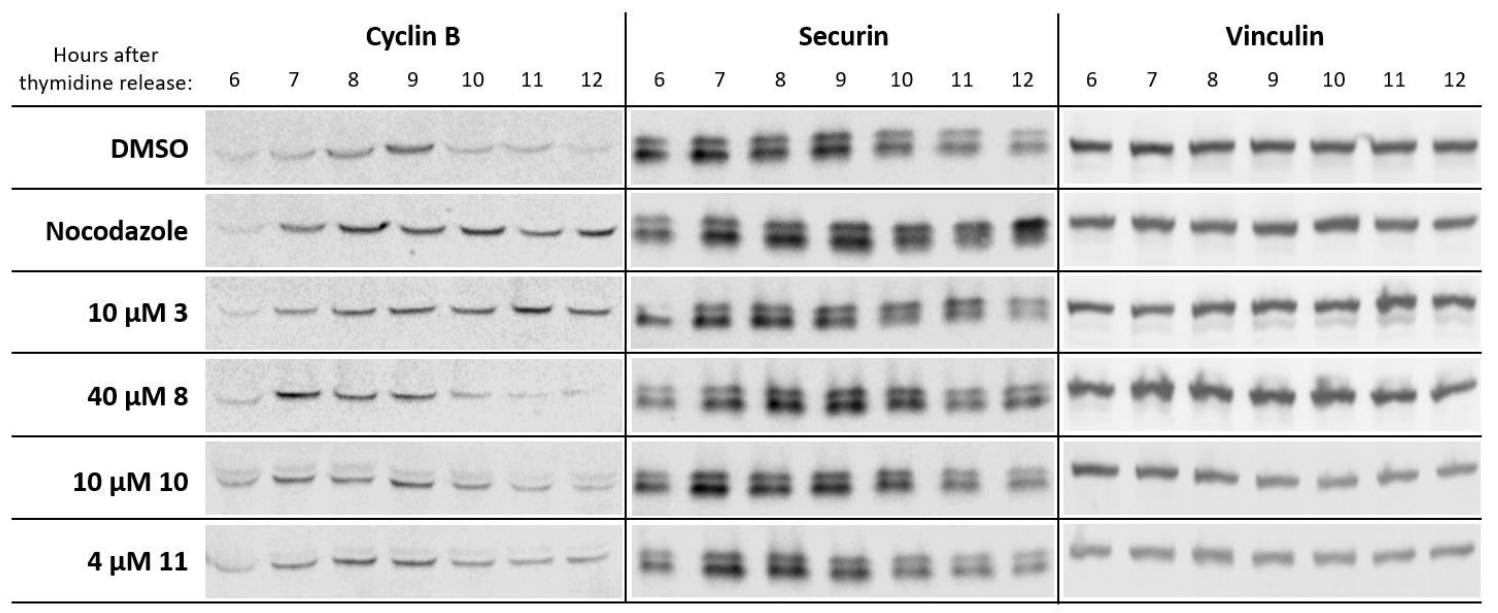

Figure 8. APC/C mitotic targets display extended kinetics of degradation when exposed to hit compounds. Western blot time course analysis of cyclin B and securin in synchronized A375 cells treated with compounds $3(10 \mu \mathrm{M}), 8(40$ $\mu \mathrm{M}), 10(10 \mu \mathrm{M})$, and $11(4 \mu \mathrm{M})$. DMSO and nocodazole $(100 \mathrm{nM})$ were used as controls. Vinculin was detected to ensure equal protein. 


\section{CELL CYCLE EFFECTS OF HIT COMPOUNDS IN A SAC-DEFICIENT MELANOMA CELL LINE}

As detailed previously, resistance to microtubule-targeting chemotherapeutics is a major issue. Many cases of resistance result from mutations in SAC proteins [113]. These mutations lead to SAC-deficient cancers, which are not arrested in mitosis in response to disruption of microtubule dynamics. Recent studies have focused on the development of drugs targeting the proteins that regulate the mitotic spindle, including the motor protein Kinesin5, Aurora kinases, and Polo-like kinases. While these drugs are less neurotoxic than taxanes, their efficacy is no better or worse against solid tumors [111]. Targeting the master regulator of mitosis, the APC/C, addresses these issues. The APC/C is essential to cell proliferation and mutations causing dysfunctional APC/C result in non-viable cells $[99,114]$. Therefore, unlike SAC inactivation, which promotes resistance, APC/C inactivating mutations should be fatal. In SAC-proficient cells, unattached kinetochores cause mitotic arrest through SAC activation, but the downstream target of the SAC is the APC/C. Therefore, SACdeficient cells should arrest in mitosis in response to APC/C inhibition because compounds targeting the APC/C bypass the need for a functional SAC.

To examine the effects of hit compounds in SAC-deficient cells, several experiments were performed in SK-MEL-28 cells, which are resistant to paclitaxel-induced mitotic arrest (Figure 9). To examine cytotoxicity of hit compounds in this cell line, colony forming assays were performed with

compounds 8, 3, and 3a1 (an analog of 3) (Figure 9A). All three compounds 

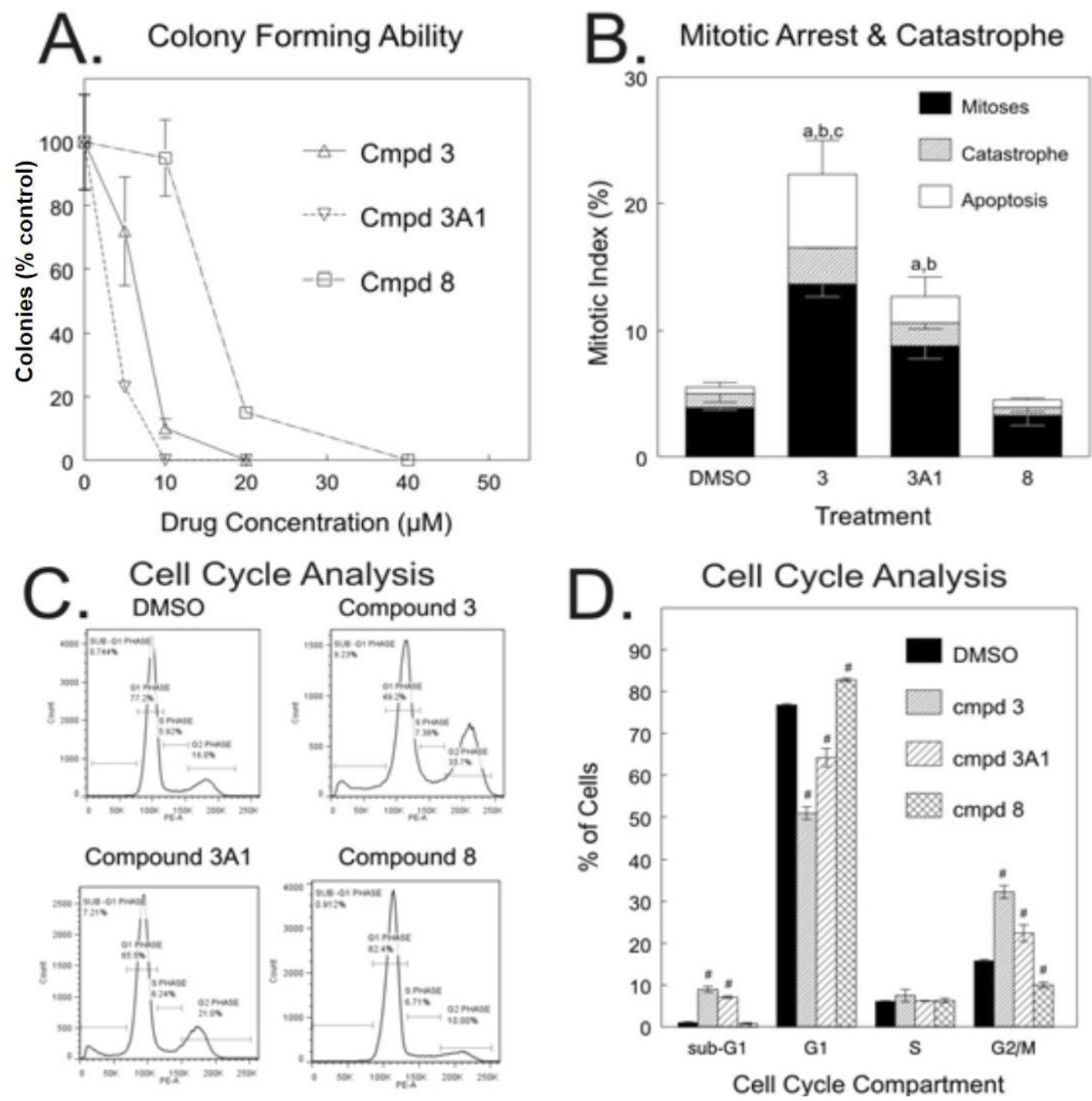

Figure 9. Compounds targeting distinct, overlapping regions of ANAPC2 induce cell cycle arrest at different points in SAC-deficient melanoma cells.

A) Colony forming assays with SK-MEL-28 cells exposed to the indicated concentrations of compounds 3, 3a1, and 8. B) Quantitation of mitoses, mitotic catastrophes, and apoptosis by $20 \mu \mathrm{M}$ compounds 3, 3a1, and 8. C) Histograms from flow cytometry of propidium iodide-stained cells exposed to $20 \mu \mathrm{M}$ compounds 3, 3a1, and 8 for 24 h. D) Quantitation of data in panel C (\# = p<0.05 vs. DMSO). Data courtesy of Brian Sils and Shuchismita Satpathy. 
dose-dependently reduced colony formation. Mitotic index analysis revealed that compounds 3 and $3 a 1$ increased the population of mitotic cells, but the mitotic index of cells treated with compound 8 was not changed (Figure 9B). Cell cycle analysis by propidium iodide staining and flow cytometry indicate that compounds 3 and 3a1 increased the population of cells in the G2/M compartment and decreased the population in G1/S, correspondingly (Figure 9C, top right panel, lower left panel). Interestingly, exposing cells to compound 8 had the opposite effect, with an increase in the G1/S compartment and a corresponding decrease in the G2/M (Figure 9C, lower right panel). The cause of this difference in cell cycle distribution induced by compounds 3 and $3 a 1$ vs. compound 8 is unclear, at this time. 


\section{APOPTOSIS ASSAYS IN LUNG AND OVARIAN CELL LINES}

Hit compounds induced mitotic arrest and cell death in multiple cancer cell types. The mechanism of cell death is unclear, but the prediction is that compounds induce apoptosis in cancer cells. To investigate the mechanism of cell death induced by hit compounds, apoptosis assays were performed to detect active caspase-3/7, a common marker of apoptosis. Malignant lung cells treated with compounds $3,8,10$ \& 11 and ovarian cell lines treated with compounds 8 , 10 \&11 displayed increased activation of apoptosis (Figure 10A, B, D, E). In contrast, non-malignant HBEC3-KT lung cells and tGM24 diploid skin fibroblast cells, showed no increase in caspase-3/7 activity, and, therefore, no apoptosis activation, when exposed to compounds $3,8,10 \& 11$ (Figure 10C, F). This result is consistent with the cytostatic effect of compounds in non-malignant cells observed in colony forming assays. 

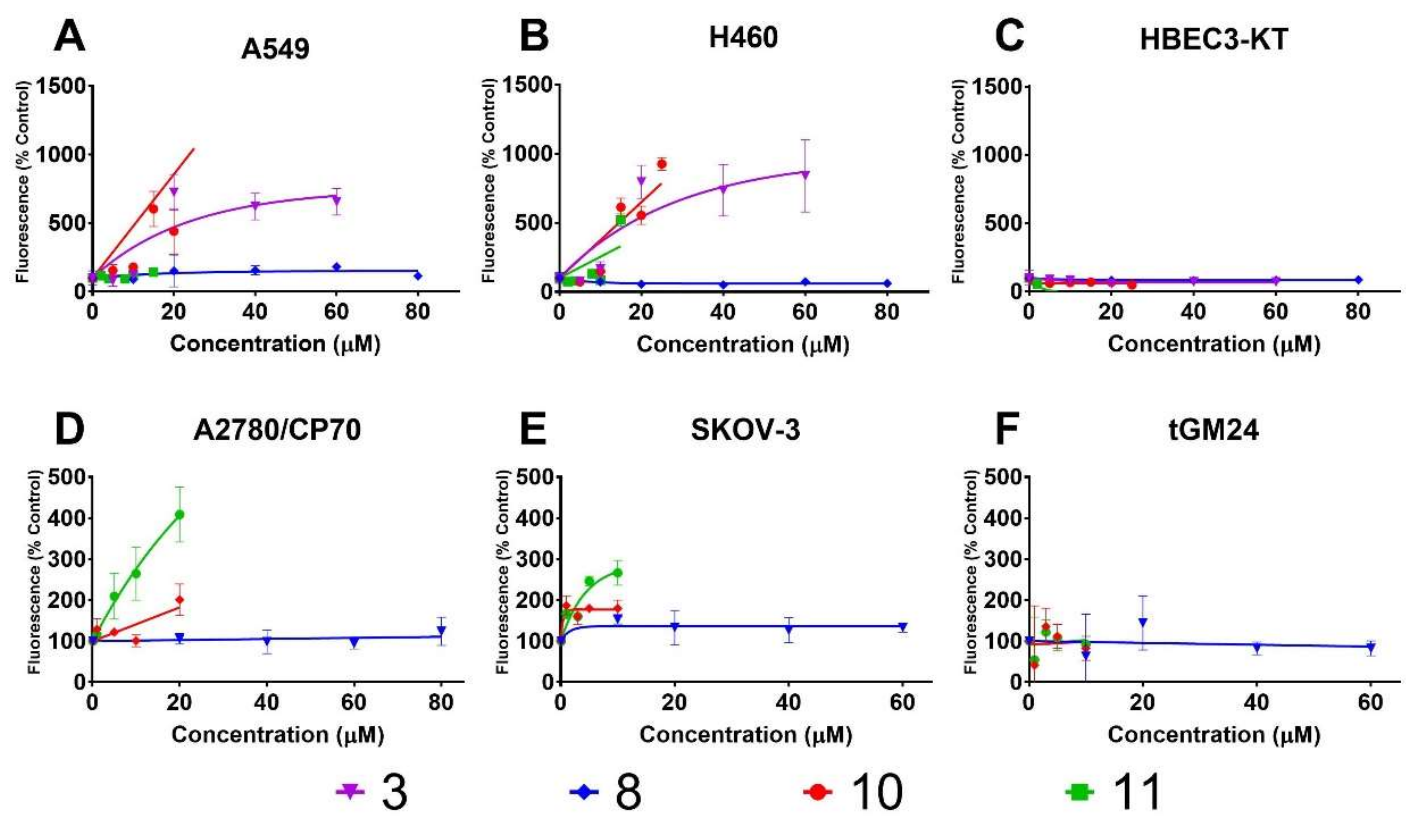

Figure 10. Levels of active caspase-3/7 are elevated in lung and ovarian cancer cells following exposure to hit compounds. Apoptosis assays were performed with compounds 8, 10, and 11 in SKOV-3 and A2780/CP70 ovarian cancer cells, and tGM24 telomerase-immortalized fibroblasts. Ovarian cells were exposed to compounds 8,10 , and 11 for $24 \mathrm{~h}$ at the indicated concentrations before caspase-3/7 activity was measured. Fibroblast cells were exposed to compounds 8,10 , and 11 for $48 \mathrm{~h}$ at the indicated concentrations before caspase-3/7 activity was measured. 


\section{DISCUSSION}

Mitotic inhibitors are critical tools in the treatment of cancer. Recently, however, numerous issues concerning current microtubule-targeting antimitotics, have received increased attention. Arguably the most significant issue with antimicrotubule drugs is the development of resistant tumors through mutations in SAC proteins. Currently, the only approved antimitotic agents target tubulin, meaning that resistant tumors are resistant to all antimitotic drugs. The work described in this thesis is significant because it provides evidence that targeting the proteins that regulate mitotic progression, namely the $A P C / C$, is a strategy with numerous potential benefits over microtubule-targeting drugs. Chief among these benefits is the fact that targeting the APC/C bypasses the need for a functional SAC, meaning that compounds inhibiting the APC/C should be effective in tumors resistant to current antimitotics. The APC/C is crucial for the metaphase-anaphase transition, but also plays significant roles in mitotic exit and G1 progression. The fact that the APC/C regulates multiple points in the cell cycle should increase the likelihood that APC/C inhibition will cause cell cycle arrest. This hypothesis is supported by results of studies with paclitaxel-resistant melanoma cells (Figure 9). Taken together, the data reported in this thesis provide evidence that further research and development of compounds targeting the APC/C will lead to a valuable new class of antimitotic agents for cancer treatment and for research into APC/C function. 
AlamarBlue, combined with colony forming data, demonstrates that hit compounds reduce the replicative ability of multiple cancer types, including lung, ovarian, pancreatic, and melanoma. This finding is consistent with the prediction that compounds inhibit the APC/C, leading to mitotic arrest and cell death. The same assays performed in SAC-deficient cells showed that the APC/C is a suitable target for the treatment of paclitaxel-resistant cancers. There was a general trend in the effective concentrations of these compounds, with compound 11 killing cells at the lowest concentrations, followed sequentially by compounds 10,3 , and 8 . However, compounds 3,8 , and 10 have a broader therapeutic window, as evidenced by colony forming assays and apoptosis assays, suggesting further development will allow improved efficacies and selectivity.

Mitotic index analysis of hit compounds in the same cell lines revealed significant increases in the population of mitotic cells, compared to DMSO. Generally, mitotic index was much greater in cells treated with paclitaxel than with hit compounds. However, direct comparisons between the efficacy of hit compounds and paclitaxel are not useful, at this early stage of development. These hit compounds contain several structural features that will allow the synthesis of analog libraries for structure-activity relationship studies (SAR) to improve efficacy.

This work also demonstrates that, to varying degrees, candidate compounds stabilize or delay the degradation of two APC/C substrates that are key to mitotic progression: cyclin B and securin. Interestingly, exposure to either compound 3 or compound 8 resulted in the opposite pattern of cyclin B and 
securin stabilization. Compound 3 completely stabilized cyclin B and delayed securin degradation, consistent with mitotic arrest and APC/C inhibition.

Compound 8 had no effect on cyclin B, but appeared to slow the kinetics of securin degradation. Degradation of securin is required for activation of separase and cleavage of cohesins, so this result suggests that cells treated with compound 8 may be exiting mitosis into a pseudo-G1 state, in which cells contain 2C DNA content. Several studies have demonstrated other mechanisms by which securin levels can be reduced, indicating that total APC/C inhibition may not result in full stabilization. Extended mitotic arrest can lead to mitotic catastrophe, a type of mitosis-linked cell death in response to chromosomal damage, and some have found that DNA damage can cause decreased expression of securin via TP53-mediated repression [115]. Therefore, if compounds cause extended mitotic arrest and mitotic catastrophe, as observed in ovarian cancer cells shown in Figure 6, total stabilization of securin may not occur. Additionally, UV radiation can cause mitotic arrest and catastrophe, and was found to lead to degradation of securin mediated by an SCF complex (SKP1-CUL1- $\beta$ TrCP) [54]. The phosphorylated, mitotic form of securin has also been found to be a target for SCF-mediated degradation [55]. These redundant mechanisms for the degradation of securin may account for some of the differences seen in its pattern of degradation. Further complicating matters, the APC/C and SCF complexes are highly similar and the homology models were generated using published structures of the SCF. Therefore, it is possible that 
compounds may be targeting one or the other or both complexes, a factor that complicates interpretations of these data.

Importantly, candidate compounds were determined to be effective in SAC-deficient, paclitaxel-resistant SK-MEL-28 cells. These cells, interestingly, were arrested at different cell cycle stages when exposed to either compound 3 or compound 8 . The mechanism to account for this difference is still unclear. However, one possibility is differences in the predicted ANAPC2 binding interactions between the two (Figure 11). 


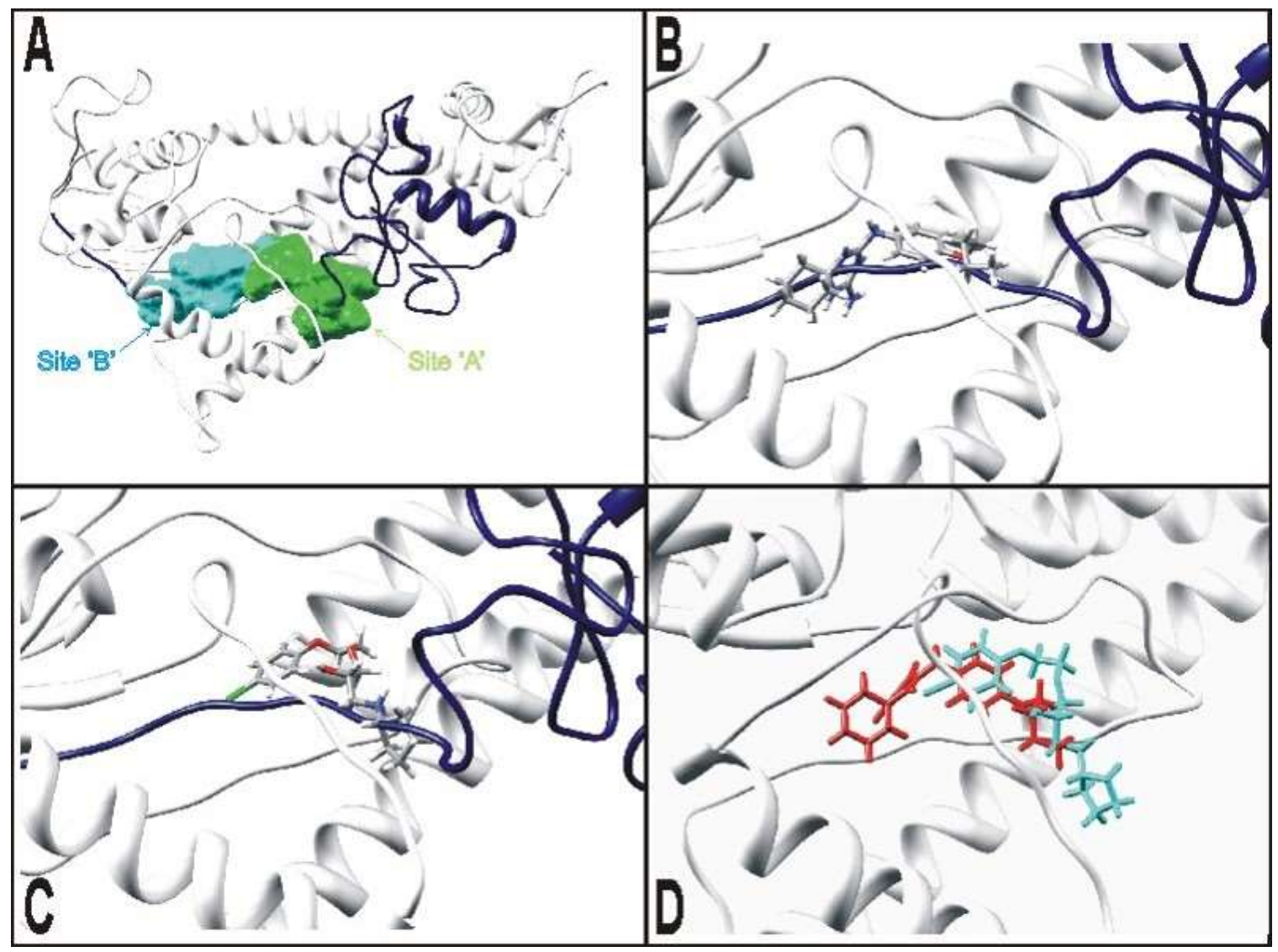

Figure 11. Two regions of ANAPC2 targeted in silico and predicted binding interactions of compounds $\mathbf{3}$ and $\mathbf{8}$. A) Homology models of the C-terminal domain of ANAPC2 (white) and ANAPC11 (blue) showing two regions of the ANAPC11-binding domain of ANAPC2 that were targeted in silico (turquoise, green). B) Predicted binding interaction of compound 3 with ANAPC2 (white) in its ANAPC11 (blue) binding domain. C) Predicted binding interaction of compound 8 with ANAPC2 (white) in its ANAPC11 (blue) binding domain. D) Overlay of the predicted binding interactions of compounds 3 (red) and 8 (turquoise) with ANAPC2 (white). 
Compound 3 is a triazospiro-containing compound, while compound 8 contains an amino-2-propanol structure. The two compounds are predicted to target distinct but overlapping sites at the ANAPC11-binding region of ANAPC2 (Figure 11B-D). It is possible that one or both compounds, rather than preventing binding of ANAPC11, alter the binding interaction. Changing the binding interaction could lead to altered, rather than inhibited, activity of the APC/C, increasing or decreasing its ability to ubiquitinylate certain substrates, leading to arrest in either mitosis or G1, depending on the effect. As mentioned previously, we cannot yet rule out the possibility that compounds inhibit SCF complexes and not the APC/C. Therefore, it is possible that these two compounds interact differently with these two E3s, leading to the observed differences in cell cycle distribution.

The activity of these compounds in SAC-deficient cells is an exciting finding, as it suggests further development may result in a class of drugs that can be used in tumors resistant to tubulin-targeting drugs. 


\section{FUTURE STUDIES}

A major issue concerning the future of this project is demonstration that compounds are specifically targeting the APC/C. While much of the data are consistent with APC/C inhibition, there are other explanations for the effects of these compounds, such as interactions with the CUL1 and RBX1 subunits of SCF complexes. Future studies will be required to demonstrate the specificity of these compounds for the APC/C. Direct binding of compounds to ANAPC2 could be demonstrated using recombinant ANAPC2 and ANAPC11 for in vitro binding reactions with hit compounds. However, inhibition of ANAPC2:ANAPC11 complex formation could also be demonstrated in cells treated with compounds by immunoprecipitation of one or the other, followed by western blots to detect the presence of its binding partner. In addition to showing that compounds prevent ANAPC2:ANAPC11 binding, it is also necessary to demonstrate that they inhibit the catalytic activity. APC/C inhibition should result in decreased ubiquitinylation of its substrates. However, previous work has shown, however, that only ANAPC11, not ANAPC2, is required for ubiquitinylation of cyclin B [108]. Others have shown that ubiquitinylation of cyclin B can be catalyzed in the presence of $\mathrm{Zn}^{2+}$ and an E2 enzyme [23]. Thus, preventing the association of rAPC11 with rAPC2 is not expected to totally abrogate cyclin B ubiquitinylation. To demonstrate inhibition of securin ubiquitinylation in vitro, reactions can be performed using recombinant ANAPC2, ANAPC11, UBE2C, and an E1 enzyme. 
Like the binding reactions, inhibition of ubiquitinylation can also be shown with cells in culture by immunoprecipitating securin, following exposure to compounds.

This study provides evidence for compound-induced stabilization of APC/C mitotic substrates in one melanoma cell line. Future work will involve further characterization of the effects on APC/C substrates in other cancer types, such as the lung, ovarian, and pancreatic cells used in this study. In addition to analysis in other cell lines, future studies will examine how compounds affect the stability of APC/C targets in G1, such as geminin, FOXM1, and CDH1. These G1 target proteins are likely stabilized in SAC-deficient cells, such as SK-MEL-28 melanoma cells.

Further work using SAC-deficient cancers is also necessary if compounds are to be demonstrated as a viable alternative to microtubule-targeting drugs. In addition to cell lines which have developed resistance to these drugs by natural means, compounds will be tested in cells that have the SAC knocked-down by SiRNA.

Additionally, developing these compounds into drug-like molecules will require further SAR studies. Finally, In vivo animal studies will be necessary to demonstrate that these compounds may be useful for the treatment of human cancers. 


\section{REFERENCES}

1. Murray, A.W., Recycling the cell cycle: cyclins revisited. Cell, 2004. 116(2): p. 221-34.

2. Diffley, J.F., Regulation of early events in chromosome replication. Curr Biol, 2004. 14(18): p. R778-86.

3. Fung, T.K. and R.Y. Poon, A roller coaster ride with the mitotic cyclins. Semin Cell Dev Biol, 2005. 16(3): p. 335-42.

4. Teixeira, L.K. and S.I. Reed, Ubiquitin ligases and cell cycle control. Annu Rev Biochem, 2013. 82: p. 387-414.

5. Glotzer, M., A.W. Murray, and M.W. Kirschner, Cyclin is degraded by the ubiquitin pathway. Nature, 1991. 349(6305): p. 132-8.

6. Komander, D. and M. Rape, The ubiquitin code. Annu Rev Biochem, 2012. 81: p. 203-29.

7. Hershko, A. and A. Ciechanover, The ubiquitin system. Annu Rev Biochem, 1998. 67: p. 425-79.

8. Hibbert, R.G., et al., E3 ligase Rad18 promotes monoubiquitination rather than ubiquitin chain formation by E2 enzyme Rad6. Proc Natl Acad Sci U S A, 2011. 108(14): p. 5590-5.

9. Dimova, N.V., et al., APC/C-mediated multiple monoubiquitylation provides an alternative degradation signal for cyclin B1. Nat Cell Biol, 2012. 14(2): p. 168-76.

10. King, R.W., et al., How proteolysis drives the cell cycle. Science, 1996. 274(5293): p. 1652-9.

11. Rieser, E., S.M. Cordier, and H. Walczak, Linear ubiquitination: a newly discovered regulator of cell signalling. Trends Biochem Sci, 2013. 38(2): p. 94102.

12. Wang, $\mathrm{H}$., et al., Role of histone H2A ubiquitination in Polycomb silencing. Nature, 2004. 431(7010): p. 873-8.

13. Kumar, Y., V. Radha, and G. Swarup, Interaction with Sug1 enables Ipaf ubiquitination leading to caspase 8 activation and cell death. Biochem J, 2010. 427(1): p. 91-104.

14. Boname, J.M., et al., Efficient internalization of MHC I requires lysine-11 and lysine-63 mixed linkage polyubiquitin chains. Traffic, 2010. 11(2): p. 210-20.

15. Yang, W.L., et al., The E3 ligase TRAF6 regulates Akt ubiquitination and activation. Science, 2009. 325(5944): p. 1134-8.

16. Kulathu, Y. and D. Komander, Atypical ubiquitylation - the unexplored world of polyubiquitin beyond Lys48 and Lys63 linkages. Nat Rev Mol Cell Biol, 2012. 13(8): p. 508-23.

17. Asher, G., N. Reuven, and Y. Shaul, 20 s proteasomes and protein degradation "by default". Bioessays, 2006. 28(8): p. 844-9.

18. Rape, M. and S. Jentsch, Taking a bite: proteasomal protein processing. Nat Cell Biol, 2002. 4(5): p. E113-6.

19. Schulman, B.A. and J.W. Harper, Ubiquitin-like protein activation by E1 enzymes: the apex for downstream signalling pathways. Nat Rev Mol Cell Biol, 2009. 10(5): p. 319-31.

20. Markson, G., et al., Analysis of the human E2 ubiquitin conjugating enzyme protein interaction network. Genome Res, 2009. 19(10): p. 1905-11.

21. Nakayama, K.I. and K. Nakayama, Ubiquitin ligases: cell-cycle control and cancer. Nat Rev Cancer, 2006. 6(5): p. 369-81.

22. Zhao, Y. and Y. Sun, Cullin-RING Ligases as attractive anti-cancer targets. Curr Pharm Des, 2013. 19(18): p. 3215-25. 
23. Tang, Z., et al., APC2 Cullin protein and APC11 RING protein comprise the minimal ubiquitin ligase module of the anaphase-promoting complex. Mol Biol Cell, 2001. 12(12): p. 3839-51.

24. Kapanidou, M., N.L. Curtis, and V.M. Bolanos-Garcia, Cdc20: At the Crossroads between Chromosome Segregation and Mitotic Exit. Trends Biochem Sci, 2017.

25. Kraft, C., et al., The WD40 propeller domain of Cdh1 functions as a destruction box receptor for APC/C substrates. Mol Cell, 2005. 18(5): p. 543-53.

26. Petroski, M.D. and R.J. Deshaies, Mechanism of lysine 48-linked ubiquitin-chain synthesis by the cullin-RING ubiquitin-ligase complex SCF-Cdc34. Cell, 2005. 123(6): p. 1107-20.

27. Rape, M. and M.W. Kirschner, Autonomous regulation of the anaphasepromoting complex couples mitosis to S-phase entry. Nature, 2004. 432(7017): p. 588-95.

28. Yamaguchi, M., et al., Cryo-EM of Mitotic Checkpoint Complex-Bound APC/C Reveals Reciprocal and Conformational Regulation of Ubiquitin Ligation. Mol Cell, 2016. 63(4): p. 593-607.

29. Wei, W., et al., Degradation of the SCF component Skp2 in cell-cycle phase G1 by the anaphase-promoting complex. Nature, 2004. 428(6979): p. 194-8.

30. Wang, R., J.L. Burton, and M.J. Solomon, Transcriptional and post-transcriptional regulation of Cdc20 during the spindle assembly checkpoint in S. cerevisiae. Cell Signal, 2017. 33: p. 41-48.

31. Wei, R., et al., Deubiquitinases in cancer. Oncotarget, 2015. 6(15): p. 12872-89.

32. Huang, X., et al., Deubiquitinase USP37 is activated by CDK2 to antagonize APC(CDH1) and promote S phase entry. Mol Cell, 2011. 42(4): p. 511-23.

33. Vodermaier, H.C., APC/C and SCF: controlling each other and the cell cycle. Curr Biol, 2004. 14(18): p. R787-96.

34. Jia, L. and Y. Sun, SCF E3 ubiquitin ligases as anticancer targets. Curr Cancer Drug Targets, 2011. 11(3): p. 347-56.

35. Eldridge, A.G. and T. O'Brien, Therapeutic strategies within the ubiquitin proteasome system. Cell Death Differ, 2010. 17(1): p. 4-13.

36. Varshavsky, A., The ubiquitin system, an immense realm. Annu Rev Biochem, 2012. 81: p. 167-76.

37. Zheng, N., Z. Wang, and W. Wei, Ubiquitination-mediated degradation of cell cycle-related proteins by F-box proteins. Int J Biochem Cell Biol, 2016. 73: p. 99110.

38. Frescas, D. and M. Pagano, Deregulated proteolysis by the F-box proteins SKP2 and beta-TrCP: tipping the scales of cancer. Nat Rev Cancer, 2008. 8(6): p. 43849.

39. Welcker, M. and B.E. Clurman, FBW7 ubiquitin ligase: a tumour suppressor at the crossroads of cell division, growth and differentiation. Nat Rev Cancer, 2008. 8(2): p. 83-93.

40. Peters, J.M., The anaphase promoting complex/cyclosome: a machine designed to destroy. Nat Rev Mol Cell Biol, 2006. 7(9): p. 644-56.

41. Peters, J.M., Emi1 proteolysis: how SCF(beta-Trcp1) helps to activate the anaphase-promoting complex. Mol Cell, 2003. 11(6): p. 1420-1.

42. Bashir, T., et al., Control of the SCF(Skp2-Cks1) ubiquitin ligase by the APC/C(Cdh1) ubiquitin ligase. Nature, 2004. 428(6979): p. 190-3.

43. Watanabe, N., et al., M-phase kinases induce phospho-dependent ubiquitination of somatic Wee1 by SCFbeta-TrCP. Proc Natl Acad Sci U S A, 2004. 101(13): p. 4419-24. 
44. Habedanck, R., et al., The Polo kinase Plk4 functions in centriole duplication. Nat Cell Biol, 2005. 7(11): p. 1140-6.

45. Cunha-Ferreira, I., et al., The SCF/Slimb ubiquitin ligase limits centrosome amplification through degradation of SAKJPLK4. Curr Biol, 2009. 19(1): p. 43-9.

46. Guderian, G., et al., Plk4 trans-autophosphorylation regulates centriole number by controlling betaTrCP-mediated degradation. J Cell Sci, 2010. 123(Pt 13): p. 2163-9.

47. Buschhorn, B.A. and J.M. Peters, How APC/C orders destruction. Nat Cell Biol, 2006. 8(3): p. 209-11.

48. Lu, D., et al., Multiple mechanisms determine the order of APC/C substrate degradation in mitosis. J Cell Biol, 2014. 207(1): p. 23-39.

49. Agarwal, R. and O. Cohen-Fix, Phosphorylation of the mitotic regulator $P d s 1 /$ securin by Cdc28 is required for efficient nuclear localization of Esp1/separase. Genes Dev, 2002. 16(11): p. 1371-82.

50. Rape, M., S.K. Reddy, and M.W. Kirschner, The processivity of multiubiquitination by the APC determines the order of substrate degradation. Cell, 2006. 124(1): p. 89-103.

51. Williamson, A., et al., Regulation of ubiquitin chain initiation to control the timing of substrate degradation. Mol Cell, 2011. 42(6): p. 744-57.

52. Lindon, C., Control of mitotic exit and cytokinesis by the APC/C. Biochem Soc Trans, 2008. 36(Pt 3): p. 405-10.

53. Castro, A., et al., The anaphase-promoting complex: a key factor in the regulation of cell cycle. Oncogene, 2005. 24(3): p. 314-25.

54. Limon-Mortes, M.C., et al., UV-induced degradation of securin is mediated by SKP1-CUL1-beta TrCP E3 ubiquitin ligase. J Cell Sci, 2008. 121(11): p. 1825-31.

55. Gil-Bernabe, A.M., et al., Protein phosphatase $2 A$ stabilizes human securin, whose phosphorylated forms are degraded via the SCF ubiquitin ligase. Mol Cell Biol, 2006. 26(11): p. 4017-27.

56. Busino, L., et al., Degradation of Cdc25A by beta-TrCP during S phase and in response to DNA damage. Nature, 2003. 426(6962): p. 87-91.

57. Jin, J., et al., SCFbeta-TRCP links Chk1 signaling to degradation of the Cdc25A protein phosphatase. Genes Dev, 2003. 17(24): p. 3062-74.

58. Minella, A.C., et al., p53 and SCFFbw7 cooperatively restrain cyclin E-associated genome instability. Oncogene, 2007. 26(48): p. 6948-53.

59. Welburn, J.P., et al., Aurora $B$ phosphorylates spatially distinct targets to differentially regulate the kinetochore-microtubule interface. Mol Cell, 2010. 38(3): p. 383-92.

60. Taylor, S. and J.M. Peters, Polo and Aurora kinases: lessons derived from chemical biology. Curr Opin Cell Biol, 2008. 20(1): p. 77-84.

61. Kwon, Y.W., et al., Pten regulates Aurora-A and cooperates with Fbxw7 in modulating radiation-induced tumor development. Mol Cancer Res, 2012. 10(6): p. 834-44.

62. Teng, C.L., et al., FBXW7 is involved in Aurora B degradation. Cell Cycle, 2012. 11(21): p. 4059-68.

63. Nakayama, K.I., S. Hatakeyama, and K. Nakayama, Regulation of the cell cycle at the G1-S transition by proteolysis of cyclin E and p27Kip1. Biochem Biophys Res Commun, 2001. 282(4): p. 853-60.

64. Malek, N.P., et al., A mouse knock-in model exposes sequential proteolytic pathways that regulate p27Kip1 in G1 and S phase. Nature, 2001. 413(6853): p. 323-7. 
65. Bornstein, G., et al., Role of the SCFSkp2 ubiquitin ligase in the degradation of p21Cip1 in S phase. J Biol Chem, 2003. 278(28): p. 25752-7.

66. Yu, Z.K., J.L. Gervais, and H. Zhang, Human CUL-1 associates with the SKP1/SKP2 complex and regulates p21(CIP1/WAF1) and cyclin D proteins. Proc Natl Acad Sci U S A, 1998. 95(19): p. 11324-9.

67. Nakayama, K.I. and K. Nakayama, Regulation of the cell cycle by SCF-type ubiquitin ligases. Semin Cell Dev Biol, 2005. 16(3): p. 323-33.

68. Bai, C., R. Richman, and S.J. Elledge, Human cyclin F. Embo j, 1994. 13(24): p. 6087-98.

69. Fung, T.K., et al., Cyclin $F$ is degraded during G2-M by mechanisms

fundamentally different from other cyclins. J Biol Chem, 2002. 277(38): p. 351409.

70. Kong, M., et al., Cyclin $F$ regulates the nuclear localization of cyclin B1 through a cyclin-cyclin interaction. Embo j, 2000. 19(6): p. 1378-88.

71. $\quad \mathrm{Li}, \mathrm{A}$. and J.J. Blow, Non-proteolytic inactivation of geminin requires CDKdependent ubiquitination. Nat Cell Biol, 2004. 6(3): p. 260-7.

72. Park, H.J., et al., Anaphase-promoting complex/cyclosome-CDH1-mediated proteolysis of the forkhead box M1 transcription factor is critical for regulated entry into S phase. Mol Cell Biol, 2008. 28(17): p. 5162-71.

73. Sivaprasad, U., Y.J. Machida, and A. Dutta, APC/C--the master controller of origin licensing? Cell Div, 2007. 2: p. 8.

74. Clijsters, L., J. Ogink, and R. Wolthuis, The spindle checkpoint, $A P C / C(C d c 20)$, and $A P C / C(C d h 1)$ play distinct roles in connecting mitosis to $S$ phase. J Cell Biol, 2013. 201(7): p. 1013-26.

75. Chang, L., et al., Atomic structure of the APC/C and its mechanism of protein ubiquitination. Nature, 2015. 522(7557): p. 450-4.

76. Zhou, Z., et al., Insights into APC/C: from cellular function to diseases and therapeutics. Cell Div, 2016. 11: p. 9.

77. Vodermaier, H.C. and J.M. Peters, APC activators caught by their tails? Cell Cycle, 2004. 3(3): p. 265-6.

78. Fang, G., H. Yu, and M.W. Kirschner, Direct binding of CDC20 protein family members activates the anaphase-promoting complex in mitosis and $\mathrm{G} 1$. Mol Cell, 1998. 2(2): p. 163-71.

79. Vodermaier, H.C. and J.M. Peters, Conspiracy to disarm APC in interphase. Nat Cell Biol, 2002. 4(5): p. E119-20.

80. Moshe, Y., et al., Role of Polo-like kinase in the degradation of early mitotic inhibitor 1, a regulator of the anaphase promoting complex/cyclosome. Proc Natl Acad Sci U S A, 2004. 101(21): p. 7937-42.

81. Hansen, D.V., et al., Plk1 regulates activation of the anaphase promoting complex by phosphorylating and triggering SCFbetaTrCP-dependent destruction of the APC Inhibitor Emi1. Mol Biol Cell, 2004. 15(12): p. 5623-34.

82. Faesen, A.C., et al., Basis of catalytic assembly of the mitotic checkpoint complex. Nature, 2017.

83. Kim, S. and H. Yu, Mutual regulation between the spindle checkpoint and APC/C. Semin Cell Dev Biol, 2011. 22(6): p. 551-8.

84. Rieder, C.L., et al., The checkpoint delaying anaphase in response to chromosome monoorientation is mediated by an inhibitory signal produced by unattached kinetochores. J Cell Biol, 1995. 130(4): p. 941-8.

85. Musacchio, A., The Molecular Biology of Spindle Assembly Checkpoint Signaling Dynamics. Curr Biol, 2015. 25(20): p. R1002-18. 
86. Kraft, C., et al., Mitotic regulation of the human anaphase-promoting complex by phosphorylation. Embo j, 2003. 22(24): p. 6598-609.

87. Lin, Z., X. Luo, and H. Yu, Structural basis of cohesin cleavage by separase. Nature, 2016. 532(7597): p. 131-4.

88. Zachariae, W., et al., Control of cyclin ubiquitination by CDK-regulated binding of Hct1 to the anaphase promoting complex. Science, 1998. 282(5394): p. 1721-4.

89. Kramer, E.R., et al., Mitotic regulation of the APC activator proteins CDC20 and CDH1. Mol Biol Cell, 2000. 11(5): p. 1555-69.

90. Qiao, X., et al., APC/C-Cdh1: From cell cycle to cellular differentiation and genomic integrity. Cell Cycle, 2010. 9(19): p. 3904-12.

91. Lindon, C. and J. Pines, Ordered proteolysis in anaphase inactivates Plk1 to contribute to proper mitotic exit in human cells. J Cell Biol, 2004. 164(2): p. 23341.

92. Zhao, W.M. and G. Fang, Anillin is a substrate of anaphase-promoting complex/cyclosome (APC/C) that controls spatial contractility of myosin during late cytokinesis. J Biol Chem, 2005. 280(39): p. 33516-24.

93. Taguchi, S., et al., Degradation of human Aurora-A protein kinase is mediated by $h$ Cdh1. FEBS Lett, 2002. 519(1-3): p. 59-65.

94. Nguyen, H.G., et al., Mechanism of Aurora-B degradation and its dependency on intact KEN and A-boxes: identification of an aneuploidy-promoting property. Mol Cell Biol, 2005. 25(12): p. 4977-92.

95. Stewart, S. and G. Fang, Destruction box-dependent degradation of aurora $B$ is mediated by the anaphase-promoting complex/cyclosome and Cdh1. Cancer Res, 2005. 65(19): p. 8730-5.

96. Singh, S.A., et al., Co-regulation proteomics reveals substrates and mechanisms of APC/C-dependent degradation. Embo j, 2014. 33(4): p. 385-99.

97. Song, L. and M. Rape, Regulated degradation of spindle assembly factors by the anaphase-promoting complex. Mol Cell, 2010. 38(3): p. 369-82.

98. Barford, D., Structural insights into anaphase-promoting complex function and mechanism. Philos Trans R Soc Lond B Biol Sci, 2011. 366(1584): p. 3605-24.

99. Zhang, J., et al., Functional characterization of Anaphase Promoting Complex/Cyclosome (APC/C) E3 ubiquitin ligases in tumorigenesis. Biochim Biophys Acta, 2014. 1845(2): p. 277-93.

100. Zirkle, R.E., Ultraviolet-microbeam irradiation of newt-cell cytoplasm: spindle destruction, false anaphase, and delay of true anaphase. Radiat Res, 1970. 41(3): p. 516-37.

101. Rieder, C.L. and R.E. Palazzo, Colcemid and the mitotic cycle. J Cell Sci, 1992. 102 ( Pt 3): p. 387-92.

102. Cheeseman, I.M., The kinetochore. Cold Spring Harb Perspect Biol, 2014. 6(7): p. a015826.

103. Howell, B.J., et al., Spindle checkpoint protein dynamics at kinetochores in living cells. Curr Biol, 2004. 14(11): p. 953-64.

104. Collin, P., et al., The spindle assembly checkpoint works like a rheostat rather than a toggle switch. Nat Cell Biol, 2013. 15(11): p. 1378-85.

105. van der Horst, A. and S.M. Lens, Cell division: control of the chromosomal passenger complex in time and space. Chromosoma, 2014. 123(1-2): p. 25-42.

106. Czuczman, N.M., et al., Pevonedistat, a NEDD8-activating enzyme inhibitor, is active in mantle cell lymphoma and enhances rituximab activity in vivo. Blood, 2016. 127(9): p. 1128-37. 
107. Swords, R.T., et al., Pevonedistat (MLN4924), a First-in-Class NEDD8-activating enzyme inhibitor, in patients with acute myeloid leukaemia and myelodysplastic syndromes: a phase 1 study. Br J Haematol, 2015. 169(4): p. 534-43.

108. Taylor, B.F., Arsenite inhibition of mitotic progression. (Ph.D. Dissertation). 2007, University of Louisville.

109. Geley, S., et al., Anaphase-promoting complex/cyclosome-dependent proteolysis of human cyclin A starts at the beginning of mitosis and is not subject to the spindle assembly checkpoint. J Cell Biol, 2001. 153(1): p. 137-48.

110. van Zon, W. and R.M. Wolthuis, Cyclin A and Nek2A: APC/C-Cdc20 substrates invisible to the mitotic spindle checkpoint. Biochem Soc Trans, 2010. 38(Pt 1): p. 72-7.

111. Huang, H.C., et al., Evidence that mitotic exit is a better cancer therapeutic target than spindle assembly. Cancer Cell, 2009. 16(4): p. 347-58.

112. London, N. and S. Biggins, Signalling dynamics in the spindle checkpoint response. Nat Rev Mol Cell Biol, 2014. 15(11): p. 736-47.

113. Yusuf, R.Z., et al., Paclitaxel resistance: molecular mechanisms and pharmacologic manipulation. Curr Cancer Drug Targets, 2003. 3(1): p. 1-19.

114. Li, M., J.P. York, and P. Zhang, Loss of Cdc20 causes a securin-dependent metaphase arrest in two-cell mouse embryos. Mol Cell Biol, 2007. 27(9): p. 34818.

115. Zhou, Y., et al., DNA damage-induced inhibition of securin expression is mediated by p53. J Biol Chem, 2003. 278(1): p. 462-70. 


\section{APPENDIX}

\section{LIST OF ABBREVIATIONS}

ANAPC1

ANAPC10

ANAPC11

ANAPC2

ANAPC2-

CTD

ANAPC3

ANAPC4

ANAPC5

ANAPC6

ANAPC8

ANOVA

APC/C

ATP

BCA

Bora

BRCA1

BUB3
Anaphase Promoting Complex Subunit 1

Anaphase Promoting Complex Subunit 10

Anaphase Promoting Complex Subunit 11

Anaphase Promoting Complex Subunit 2

Anaphase Promoting Complex Subunit 2 C-terminal Domain

Anaphase Promoting Complex Subunit 3

Anaphase Promoting Complex Subunit 4

Anaphase Promoting Complex Subunit 5

Anaphase Promoting Complex Subunit 6

Anaphase Promoting Complex Subunit 8

Analysis of Variance

Anaphase Promoting Complex/ Cyclosome

Adenosine Triphosphate

Bicinchoninic Acid

Protein Aurora Borealis

Breast Cancer Type 1 Susceptibility Protein

Budding Uninhibited by Benzimidazoles 3 


\begin{tabular}{ll} 
BUBR1 & Budding Uninhibited by Benzimidazoles 1: BUB1B \\
CDC20 & Cell Division Cycle 20 \\
CDC25A & Cell Division Cycle 25A \\
CDH1 & CDC20 Homolog 1 \\
CDK & Cyclin-dependent Kinase \\
CDK1 & Cyclin-dependent Kinase 1 \\
CDKN & Cyclin-dependent Kinase Inhibitor \\
CDKN1A & Cyclin-dependent Kinase Inhibitor 1A \\
CDKN1B & Cyclin-dependent Kinase Inhibitor 1B \\
CDKN1C & Cyclin-dependent Kinase Inhibitor 1C \\
CDT1 & Chromatin Licensing and DNA Replication Factor 1 \\
CDT2 & Chromatin Licensing and DNA Replication Factor 2 \\
CENP-A & Histone H3-like Centromeric Protein A \\
CFE & Colony Forming Efficiency \\
CHK1 & Checkpoint Kinase-1 \\
CRL & Cullin-RING Ligase \\
C-terminus & Carboxy-terminus \\
CUL1 & Cullin-1 \\
DMSO & Dimethyl Sulfoxide \\
DNA & Deoxyribonucleic Acid \\
DUB & Deubiquitinating Enzyme \\
E1 & Ubiquitin-activating Enzyme \\
E2 & Ubiquitin-conjugating Enzyme \\
E3 & Ubitin Ligase \\
\hline
\end{tabular}




$\begin{array}{ll}\text { ECL } & \text { Enhanced Chemiluminescence } \\ \text { EDTA } & \text { Ethylenediaminetetraacetic Acid } \\ \text { EGF } & \text { Epidermal Growth Factor } \\ \text { EMI1 } & \text { Early Mitotic Inhibitor 1 } \\ \text { FBXL } & \text { F-box/ LRR-repeat Protein } \\ \text { FBXL1 } & \text { F-box/ LRR-repeat Protein 1 } \\ \text { FBXO } & \text { F-box Only Protein } \\ \text { FBX01 } & \text { F-box Only Protein 1 } \\ \text { FBX05 } & \text { F-box Only Protein 5 } \\ \text { FBXW } & \text { F-box/ WD Repeat-containing protein } \\ \text { FBXW1 } & \text { F-box/ WD Repeat-containing protein 1 } \\ \text { FBXW7 } & \text { F-box/ WD Repeat-containing protein 7 } \\ \text { FOXM1 } & \text { Forkhead Box Protein M1 } \\ \text { G1 } & \text { Gap 1 } \\ \text { G2 } & \text { Gap 2 } \\ \text { HECT } & \text { Homologous to E6-AP C-terminus } \\ \text { KIFC1 } & \text { Kinesin Family Member C1 } \\ \text { KMN } & \text { Kitotic Arrest-deficient 2 } \\ \text { KNL1 } & \text { KNL1-MIS12-NDC80 Network } \\ \text { LRR } & \text { Mitosis } \\ \text { M } & \text { Mitotic Arrest-deficient 1 } \\ \text { MAD1 } & \text { MAD2 }\end{array}$




\begin{tabular}{ll} 
MDM2 & Mouse Double Minute 2 \\
MELT & Met-Glu-Leu-Thr \\
MPS1 & Monopolar Spindle 1 Kinase \\
MTT & $\begin{array}{l}\text { 3-(4,5-dimethylthiazol-2-yl)-2,5-diphenyltetrazolium } \\
\text { bromide }\end{array}$ \\
NEDD8 & $\begin{array}{l}\text { Neural Precursor Cell Expressed Developmentally Down } \\
\text { regulated Protein 8 }\end{array}$ \\
NEK2A & Never in Mitosis Gene A-related Kinase 2 \\
N-terminus & Amino-terminus \\
PBS & Phosphate-buffered Saline \\
PDB & Protein Database \\
PHD & Plant Homeodomain \\
PLK1 & Polo-like Kinase 1 \\
PLK4 & Polo-like Kinase 4 \\
pre-RC & Pre-replication Complex \\
PTM & Post-translational Modifications \\
RAD21 & Double-strand-break Repair Protein rad21 Homolog \\
RBX1 & RING-box Protein 1 \\
RING & Really Interesting New Gene \\
RNA & Ribonucleic Acid \\
S & Synthesis, DNA Replication \\
SAC & Spindle Assembly Checkpoint \\
SAR & Structure Activity Relationship \\
SCF & SKP/Cullin/F-box-containing Complexes \\
SiRNA & Smterfering RNA \\
\hline
\end{tabular}


SKP1

SKP2

TBST

TP53

TPR

UBE2C

UPS

USP37

UV

WEE1

Zn

$\beta-T r C P$
S-phase Kinase-associated Protein 1

S-phase Kinase-associated Protein 2

Tris-buffered Saline with Tween $\circledR 20$

Tumor Protein P53

Tetratricopeptide Repeat

Ubiquitin-conjugating Enzyme E2 C

Ubiquitin-proteasome System

Ubiquitin Carboxyl-terminal Hydrolase 37

Ultraviolet

Wee1-like Protein Kinase

Zinc

Beta-transducin Repeat Containing Protein 


\section{CURRICULUM VITAE}

J. Mason Hoffman

\section{CONTACT INFORMATION:}

Home Address: 832 E. Madison St. Apt. A

Louisville, KY 40204

Telephone:

(859) 274-2876

E-mail Address: jmhoff08@louisville.edu

EDUCATION:

$2010-2014$ Sewanee: The University of the South; Sewanee, TN; B.S. in Biochemistry

2014 -Present $\quad$ University of Louisville; Louisville, KY; Graduate Student in Pharmacology and Toxicology

HONORS \& AWARDS:

$2014-2016 \quad$ IPIBS Fellowship, University of Louisville

2015 Best poster presentation, Masters Student. Ohio Valley Society of Toxicology 2015 Annual meeting. Northern

Kentucky University, Highland Heights, KY

PROFESSIONAL SOCIETY MEMBERSHIPS

2016

American Society for Pharmacology and Experimental

Therapeutics (ASPET)

PUBLICATIONS:

Articles published in refereed journals: 
1. Preparation of C-arylglycals via Suzuki-Miyaura cross-coupling of dihydropyranylphosphates. Leidy, M. R.; Hoffman, J. M.; Pongdee, R. Tetrahedron Lett. 54(50): 6889-6891 (2013)

2. Synthesis of N,N-Diethylbenzamides via a Non-Classical Mitsunobu Reaction. Hoffman, J. M.; Miller, J. N.; Gardner, M. E.; LePar, D. R.; Pongdee, R. Synth. Commun. 44(7): 976-980 (2014)

Abstracts:

1. Purification of the C-terminal Domain of ANAPC2 and Evidence Supporting the Interaction of Lead Compounds for Inhibition of Mitosis. Hoffman, J. M.; Al-Eryani, L.; Saforo, D. J.; Taylor, B. F.; Trent, J. O.; Garbett, N. C.; States, J. C., FASEB J. April 2016 30:Ib474

\section{PRESENTATIONS:}

1. Synthesis of N,N-Diethylbenzamides via a Non-Classical Mitsunobu Reaction. Hoffman, J. M.; Miller, J. N.; Gardner, M. E.; LePar, D. R.; Pongdee, R. 43 ${ }^{\text {rd }}$ National Organic Chemistry Symposium, June 2013, University of Washington, Seattle, WA. (Poster)

2. Preparation of C-Arylglycals via the Suzuki-Miyaura Cross-Coupling Reaction of Cyclic Ketene Acetal Phosphates. Leidy, M. R.; Hoffman, J. M.; Pongdee, R. 43 ${ }^{\text {rd }}$ National Organic Chemistry Symposium, June 2013, University of Washington, Seattle, WA. (Poster)

3. Construction of N,N-Diethylbenzamides Employing a Non-Classical Mitsunobu Reaction. Hoffman, J. M.; Miller, J. N.; Gardner, M. E.; LePar, D. R.; Pongdee, R. 65th Southeast Regional American Chemical Society Meeting, November 2013, Atlanta, GA. (Poster)

4. New Methods for C-Arylglycal Construction: Suzuki-Miyaura CrossCoupling of Dihydropyranylphosphates. Leidy, M. R.; Hoffman, J. M.; Pongdee R. 65th Southeast Regional American Chemical Society Meeting, November 2013, Atlanta, GA. (Poster)

5. Inhibiting the Anaphase-Promoting Complex/Cyclosome: An Innovative Approach for Cancer Chemotherapy. Udoh, K. T.; Hoffman, J. M.; Trent, J. O.; States, J. C., Research!Louisville, November 2015. University of Louisville, Louisville, KY. (Poster)

6. Purification of the C-terminal Domain of ANAPC2 and Evidence Supporting the Interaction of Lead Compounds for Inhibition of Mitosis. Hoffman, J. M.; Al-Eryani, L.; Saforo, D. J.; Taylor, B. F.; Trent, J. O.; Garbett, N. C.; States, J. C., Research!Louisville, November 2015. University of Louisville, Louisville, KY. (Poster)

7. Purification of the C-terminal Domain of ANAPC2 and Evidence Supporting the Interaction of Lead Compounds for Inhibition of Mitosis. Hoffman, J. M.; Al-Eryani, L.; Saforo, D. J.; Taylor, B. F.; Trent, J. O.; Garbett, N. C.; States, J. C., Ohio Valley Society of Toxicology, November 2015. Northern Kentucky University, Highland Heights, KY. (Poster)

8. Purification of the C-terminal Domain of ANAPC2 and Evidence Supporting the Interaction of Lead Compounds for Inhibition of Mitosis. 
Hoffman, J. M.; Al-Eryani, L.; Saforo, D. J.; Taylor, B. F.; Trent, J. O.; Garbett, N. C.; States, J. C., Experimental Biology 2016, April 2016. San Diego, CA. (Poster)

9. Purification of the C-terminal Domain of ANAPC2 and Evidence Supporting the Interaction of Lead Compounds for Inhibition of Mitosis. Hoffman, J. M.; Al-Eryani, L.; Saforo, D. J.; Taylor, B. F.; Trent, J. O.; Garbett, N. C.; States, J. C., ASPET 2016 Annual Meeting, April 2016. San Diego, CA. (Poster)

10. Recombinant ANAPC2 Displays Multiple Internal Start Sites and Compounds targeting ANAPC2 Decrease Proliferation of Lung Cancer Cells in vitro. Hoffman, J. M.; Wainscott N. W.; Taylor B. F.; Trent J. O.; States J. C., Research!Louisville 2016, October 2016. Louisville, KY (Poster)

11. Recombinant ANAPC2 Displays Multiple Internal Start Sites and Compounds targeting ANAPC2 Decrease Proliferation of Lung Cancer Cells in vitro. Hoffman, J. M.; Wainscott N. W.; Taylor B. F.; Trent J. O.; States J. C., OVSOT 2016, October 2016. Indianapolis, IN (Poster)

12. Compounds Targeting the APC/C Induce Mitotic Arrest and Apoptosis in Cancer Cells in vitro. Hoffman, J. M., James Graham Brown Cancer Center Colloquia on Cancer Biology and Therapeutics, March 2017. Louisville, KY (Seminar)

13. Structure-Activity Relationship Studies of Oxypropanolamines: The Importance of Hydrophobicity About the Amine for Maintaining AntiProliferative Effects in Lung Cancer Cells. Hoffman, J. M.; Wainscott, N. W.; Taylor, B. F.; Burlison, J. A.; Trent, J. O.; States, J. C., Experimental Biology 2017, April 2017. Chicago, IL (Poster) 